\title{
Growth mode and electronic structure of the epitaxial $\mathrm{C}_{60}(111) / \mathrm{GeS}(001)$ interface
}

\author{
G. Gensterblum, K. Hevesi, B.-Y. Han, L.-M. Yu, J.-J. Pireaux, P. A. Thiry, \\ R. Caudano, and A.-A. Lucas \\ Institute for Studies in Interface Sciences, Facultés Universitaires Notre-Dame de la Paix, B-5000 Namur, Belgium \\ D. Bernaerts, S. Amelinckx, and G. Van Tendeloo \\ Rijksuniversitair Centrum Antwerpen, Groenenborgerlaan 171, B-2020 Antwerpen, Belgium \\ G. Bendele, T. Buslaps, and R. L. Johnson \\ II. Institut für Experimentalphysik, Universität Hamburg, Luruper Chaussee 149, D-22761 Hamburg, Germany \\ M. Foss \\ Institute of Physics and Astronomy, Aarhus University, DK-8000 Aarhus C, Denmark
}

R. Feidenhans'1

Department of Solid State Physics, Risø National Laboratory, DK-4000 Roskilde, Denmark

G. Le Lay

Centre de Recherche sur les Mécanismes de la Croissance Cristalline, Campus de Luminy, Case 913, F-13288 Marseille Cédex 9, France

(Received 31 May 1994)

The heteroepitaxial growth of $\mathrm{C}_{60}$ on $\mathrm{GeS}(001)$ has been studied using low-energy electron diffraction, selected-area electron diffraction, high-resolution electron microscopy, $x$-ray diffraction, and $x$-ray and ultraviolet-photoelectron spectroscopy (UPS). The simultaneous observation of diffraction spots characteristic of the substrate and the $\mathrm{C}_{60}(111)$ overlayer allows us to specify the geometry of the epitaxy. The shape of the intensity curves of the $\mathrm{C} 1 s$ and Ge $3 d$ photoemission lines strongly suggests a layer-bylayer-type growth, confirmed by the observation in the synchrotron $\mathrm{x}$-ray diffraction spectrum of finitesize oscillations on the (111) Bragg reflection peak of a thin $\mathrm{C}_{60}(111)$ film. From a theoretical simulation of the $\mathrm{C} 1 s$ and Ge $3 d$ line-intensity curves, the mean free path of a $\mathrm{C} 1 s$ and $\mathrm{Ge} 3 d$ photoelectron in solid $\mathrm{C}_{60}$ is estimated to about 15.4 and $17 \AA$, respectively. The plot of the film thickness versus deposition time shows evidence for a small difference in sticking coefficient between the first monolayer and the upper ones. A detailed analysis of the $\mathrm{C} 1 s$ line shapes for normal and grazing emission suggests the existence of inequivalent carbon sites at the interface. The first valence-band feature of the substrate presents a downward band bending of about $200 \mathrm{meV}$ with increasing $\mathrm{C}_{60}$ coverage. From the shift of the cutoff in the UPS spectra we deduce a work function increase of about $100 \mathrm{meV}$ upon monolayer adsorption. The characteristic spectral features of $\mathrm{C}_{60}$ observed in the UPS spectra for bulk fullerite are slightly broadened and shifted to lower binding energies at submonolayer coverages and show no direct evidence for significant hybridization, indicating that the $\mathrm{C}_{60}$-substrate interaction is mainly dominated by van der Waals bonding. All these observations can be explained by a positive effective dipole of about $8 \times 10^{-31} \mathrm{Cm}$ induced on the $\mathrm{C}_{60}$ molecule upon adsorption onto the GeS substrate.

\section{INTRODUCTION}

Over the last few years, the structural, vibrational, and electronic properties of $\mathrm{C}_{60}$ fullerene and fullerite have been studied extensively by various techniques. The availability of single-crystalline films is important for investigating in detail the solid-state properties of fullerenes. The growth of well-ordered epitaxial $\mathbf{C}_{60}$ thin films on semiconductors and metals under the welldefined conditions of molecular beam deposition has therefore attracted much interest. Good single-domain epitaxy of $\mathrm{C}_{60}$ has been reported on $\mathrm{Cu}(111),{ }^{1-3} \mathrm{Au}(110),{ }^{4}$ and $\mathrm{Ni}(110) .^{5}$ Double- or even multi-domain growth has been reported on several other metal substrates, in particular, $\mathrm{Au}(111),{ }^{6-8} \mathrm{Ag}(111),{ }^{7} \mathrm{Cu}(100),{ }^{1}$ and $\mathrm{Cu}(110) .{ }^{1}$ However, the lattice mismatch between the substrate and the $\mathrm{C}_{60}$ lattice leads to strained or slightly deformed hexagonal monolayers, ${ }^{4,9}$ the magnitude of the deformation depending on the strength of the $\mathrm{C}_{60}$-substrate interaction. In some cases, this interaction leads to the lifting of the reconstruction of the substrate surface ${ }^{6}$ or to the formation of new interface reconstructions. ${ }^{8}$ Furthermore, the domain size of these $\mathrm{C}_{60}$ overlayers is limited by the relatively high step density on metal surfaces. For instance, the domain size is about $100 \AA$ for $\mathrm{C}_{60} / \mathrm{Cu}(111),{ }^{1}$ and 500 $\AA$ for $\mathrm{C}_{60} / \mathrm{Ag}(111)^{6}$ 
Classical semiconductors like $\mathrm{Si}$ and $\mathrm{GaAs}$ have a lower step density, but on these substrates $\mathrm{C}_{60}$ forms either multidomain islands ${ }^{10-14}$ or small-grained crystalline islands of random orientation with respect to the substrate lattice. $^{16-18}$ This is a general consequence of the lattice mismatch and, in the case of $\mathrm{Si}$, of the strong adsorbate-substrate bonding, which limits the surface mobility of the $\mathrm{C}_{60}$ molecules. Therefore well-ordered films are in general obtained only at high temperatures. ${ }^{14,15,17}$ Room-temperature deposition leads to irregular thin-film growth with substantial imperfections. For instance, $\mathrm{C}_{60}$ growth on GaAs(110) leads at room temperature to the formation of commensurate first-layer islands that show distorted close-packed structures with two distinct adsorption sites. ${ }^{13}$ Multilayer growth on this strained and lattice-mismatched first monolayer produces tilted close-packed structures with small grains, defects, dislocations, stacking faults, and faceting. ${ }^{13}$ On the other hand, $\mathrm{C}_{60}$ growth at higher substrate temperatures results in the formation of a nearly perfect close-packed doubledomain film extending over thousands of angstroms. ${ }^{14,15,17}$ Single-domain films could be grown on a GaAs(110) surface with a high step density in the [112] direction, but the grain size was then again limited by the small size of the substrate terraces. ${ }^{15}$

$\mathrm{C}_{60}$ growth on $\mathrm{Si}$ has been revealed to be even more complex than on GaAs due to the stronger moleculesubstrate interaction. ${ }^{10-12}$ While multilayer $\mathrm{C}_{60}$ desorbs at $500 \mathrm{~K}$, Hamza and Balooch ${ }^{12}$ showed that first-layer $\mathrm{C}_{60}$ adsorbed on $\mathrm{Si}(100)-2 \times 1$ decomposes at $900 \mathrm{~K}$ into a graphite layer before desorbing at $1150 \mathrm{~K}$. Wang et al. ${ }^{10}$ observed two types of local ordering configurations for the first monolayer: a $c(4 \times 4)$ square-type arrangement with a nearest-neighbor distance (NND) of $10.9 \AA$ and a $c(4 \times 3)$ quasihexagonal structure with a compressed and relaxed NND of, respectively, 9.6 and $11.5 \AA$. A transition to ordered, close-packed, but not completely relaxed (NND of $10.4 \AA$ ) islands is observed for multilayer deposition. The islands present multiple layers, indicating a Stranski-Krastanov growth, and several defects as well as (100) facets. $\mathrm{C}_{60}$ deposition on $\mathrm{Si}(111)-1 \times 1$ (Ref. 19) and Si(111)-7 $\times 7$ (Refs. 11,16,17,20) has also been studied. An $\mathrm{X}$-ray diffraction study by Tong et al. ${ }^{19}$ revealed a $(3 \sqrt{3} \times 3 \sqrt{3}) R 30^{\circ}$ reconstruction with close-packed $\mathrm{C}_{60}$ molecules stacked parallel to the $\mathrm{Si}(111)-1 \times 1$ surface but with a correlation length smaller than $200 \AA$ A. Weaver and co-workers ${ }^{16,17}$ could image repeatedly isolated $\mathrm{C}_{60}$ molecules on the $\mathrm{Si}(111)-7 \times 7$ surface (indicating strong bonding) and observed no preferential bonding at step edges. $\mathrm{Xu}$, Chen, and Creager ${ }^{11}$ observed no long-range order for monolayer coverages on $\mathrm{Si}(111)-7 \times 7$, but rather a disorder-to-order transition with two preferential island orientations for multilayers as a result of a delicate balance between the intermolecular and the moleculesubstrate interactions.

As a consequence of the strong bonding on the $\mathrm{Si}(100)-2 \times 1$ surface the free rotation of the molecule is significantly impeded and scanning tunneling microscopy (STM) images reveal internal molecular structures for the first $\mathrm{C}_{60}$ monolayer. ${ }^{10}$ These internal structures reflect the partial charge density of the molecular states near the
Fermi level. Their twofold symmetry indicates adsorption with a single or double carbon bond facing the substrate. On the contrary, the threefold symmetry observed in the intramolecular structure of $\mathrm{C}_{60}$ molecules adsorbed on $\mathrm{Cu}(111)$ (Refs. 2,3) allows one to conclude that a hexagonal ring faces the substrate. Internal molecular contrast has been reported on some other substrates. ${ }^{7,21-23}$

Several recent studies ${ }^{1,4,7,10,12,24-31}$ have addressed the character of the overlayer-substrate interaction, which is an important issue in thin-film growth. It has been shown that on metals ${ }^{1,4,7,24-29}$ the first monolayer is chemisorbed due to the hybridization between $\mathrm{C}_{60} p$ and metal $d$ states, with the possibility of charge transfer. The electronic structure of the first monolayer is therefore altered with respect to the second one, which itself is essentially similar to the bulk of the film. ${ }^{24,26}$ Perturbation of the vibrational structure of first-monolayer $\mathrm{C}_{60}$ on metals has also been reported. Surface-enhanced Raman spectroscopy of $\mathrm{C}_{60}$ on $\mathrm{Au}$ (Ref. 30) and $\mathrm{Ag}$, ${ }^{31}$ and highresolution electron-energy-loss spectroscopy (HREELS) of $\mathrm{C}_{60}$ on $\mathrm{Rh}(111),{ }^{27}$ show a vibrational spectra richer than for thick films. The observation of additional vibration modes is attributed to a lowering of the $\mathrm{C}_{60}$ symmetry as a result of the overlayer-substrate interaction.

The problems encountered in growing high-quality epitaxial $\mathrm{C}_{60}$ films on metals and semiconductors can be overcome by using layered materials as substrates. ${ }^{18}$ The low surface energy of these materials and the absence of unsaturated chemical bonds at the surface relaxes the stringent lattice-matching conditions required in most cases for epitaxial growth and favors surface diffusion. Indeed, successful single-domain epitaxy has already been reported on the layered substrates $\mathrm{MoS}_{2},{ }^{32-34} \mathrm{GaSe},{ }^{34,35}$ $\mathrm{GeS},{ }^{18,36}$ and mica. ${ }^{37-40}$ Furthermore, the cleaved surface of a layered semiconductor presents a very slow step density with flat substrate terraces, several micrometers in size, which allow the growth of very large $\mathrm{C}_{60}$ islands. Island diameters of up to $1 \mu \mathrm{m}$ have been reported for $\mathrm{C}_{60} / \mathrm{MoS}_{2} \cdot{ }^{38}$

In this paper, we report on a detailed study of the formation of the epitaxial $\mathrm{C}_{60}(111) / \mathrm{GeS}(001)$ interface by several microscopic and spectroscopic techniques. The geometry of the epitaxy has been determined from lowenergy electron diffraction (LEED) and selected-area electron diffraction (SAED). High-resolution electron microscopy reveals close-packed $\mathrm{C}_{60}(111)$ islands extending over several micrometers with a low density of defects and a domain diameter limited by the size of the substrate terraces. The evolution of the $\mathrm{x}$-ray photoelectron spectroscopy (XPS) core-level intensities and the observation of finite-size oscillations on the (111) $\mathrm{C}_{60}$ Bragg reflection in the synchrotron $\mathrm{x}$-ray diffraction spectrum demonstrate that the film grows layer by layer. The electronic structure of the interface has been investigated by ultraviolet-photoelectron spectroscopy (UPS) and XPS. A detailed XPS core-level line-shape analysis together with an increase of the work function and a downward band bending observed in the substrate valence band indicate the formation of a small surface dipole. This interface dipole results from two contributions: an image- 
charge-induced dipole and an additional small charge delocalization of the $\mathrm{C}_{60}$ electrons over the substrate due to the ionic character of the latter.

\section{EXPERIMENT}

The XPS and UPS experiments have been performed with an ESCA-300 spectrometer (Scienta) equipped with an additional uv source (Leybold-Heraeus UVS 10/35). By selecting pass energies from 20 up to $1000 \mathrm{eV}$ and entrance slit widths from 0.1 up to $4.0 \mathrm{~mm}$ the instrumental resolution can be varied from 0.27 to $5 \mathrm{eV}$. The typical energy resolution in our XPS experiments is $0.29 \pm 0.01$ $\mathrm{eV}$. In the UPS mode we use a pass energy of $10 \mathrm{eV}$ and an entrance slit width of $0.2 \mathrm{~mm}$, yielding a typical instrumental resolution of about $150 \mathrm{meV}$, measured on the sharp Fermi edge of a clean tantalum foil.

The preparation chamber of the Scienta 300 is equipped with a rear-view LEED diffractometer (SpectaLEED from Omicron) using an axial electron gun with an $\mathrm{LaB}_{6}$ filament. The base pressures in the preparation and analysis chambers were $1 \times 10^{-9}$ and $2 \times 10^{-10} \mathrm{mbar}$, respectively. For our experiments we used chromatographically purified $\mathrm{C}_{60}$ (purity $>99.9 \%$ ) provided by $\mathrm{G}$. Sawatzky or purchased from Stephan Kaesdorf Geräte für Forschung und Industrie (Germany). The powder was loaded into the graphite crucible of a Knudsen cell (WA technology, UK) and carefully outgassed for $12 \mathrm{~h}$ at a temperature of $380^{\circ} \mathrm{C}$ and for $1 \mathrm{~h}$ at $420^{\circ} \mathrm{C}$. It was then sublimed at a temperature of $420^{\circ} \mathrm{C}$ at constant pressures below $2 \times 10^{-9} \mathrm{mbar}$ on a freshly in situ cleaved undoped $\mathrm{GeS}(001)$ substrate $\left(7 \times 7 \mathrm{~mm}^{2}\right)$ held at about $180^{\circ} \mathrm{C}$. The outstanding crystallographic quality of the clean substrate and of the thin $C_{60}$ (111) film was checked by LEED. The thickness of the film was either monitored by a quartz oscillator or evaluated from the intensity ratio of $\mathrm{Ge} 3 d$ and $\mathrm{C} 1 s$ core-level lines. The evaporation rate was about $0.5 \AA / \mathrm{min}$.

Samples for transmission electron microscopy (TEM) examinations from plane-view directions were prepared by cleaving a $\mathrm{GeS}(001)$ substrate covered with a $200-\AA$ thick $\mathrm{C}_{60}$ film with a razor blade along a plane parallel to the substrate/film interface plane. The thickness of the film plus substrate after $e x$ situ cleavage was of the order of a few tens of micrometers. The sample was then mounted on a copper ring and ion milled in a liquidnitrogen cooling stage. The sample was thinned only from the substrate side. A $4 \mathrm{kV}$ voltage and a $12^{\circ}$ angle of incidence with respect to the sample surface were adopted until an electron perforation was obtained in the central region of the sample.

Selected-area electron diffraction was performed in a Philips CM20 transmission electron microscope operating at $200 \mathrm{kV}$ and allowing a $\pm 45^{\circ}$ tilting angle of the specimen. High-resolution electron microscopy (HREM) was carried out in a Jeol 4000 EX transmission electron microscope operating at $400 \mathrm{kV}$ incident beam energy. Care was taken to work at low beam intensity in order to avoid irradiation damage as much as possible.

The synchrotron $x$-ray diffraction measurements were performed at the Hamburg Synchrotron Radiation Labo- ratory (HASYLAB) on the vertical diffractometer at the wiggler beamline BW2 which is specially designed for surface $\mathrm{x}$-ray diffraction studies. The instrumental resolution was $0.06^{\circ}$ full width at half maximum (FWHM), corresponding to a reciprocal space resolution of $\Delta q=0.005 \AA^{-1}$ for $\lambda=1.5216 \AA$. The thin $\mathrm{C}_{60}$ films on $\mathrm{GeS}(001)$ were prepared on the Seya-Namioka beamline as described above and were then transferred in air to the BW2 station.

\section{GEOMETRY OF THE EPITAXY}

\section{A. LEED}

Figure 1 shows LEED patterns obtained with a primary energy of $35.6 \mathrm{eV}$ before and after 6,12 , and $18 \mathrm{~min}$ of sublimation at $T_{\text {cell }}=420^{\circ} \mathrm{C}$ with the substrate held at around $180^{\circ} \mathrm{C}$. As we will explain in the next section, from the analysis of the XPS intensities it comes out that Figs. 1(b), 1(c), and 1(d) correspond to coverages of $\frac{1}{3}, \frac{2}{3}$, and $1 \mathrm{ML}$, respectively. The LEED patterns revealing
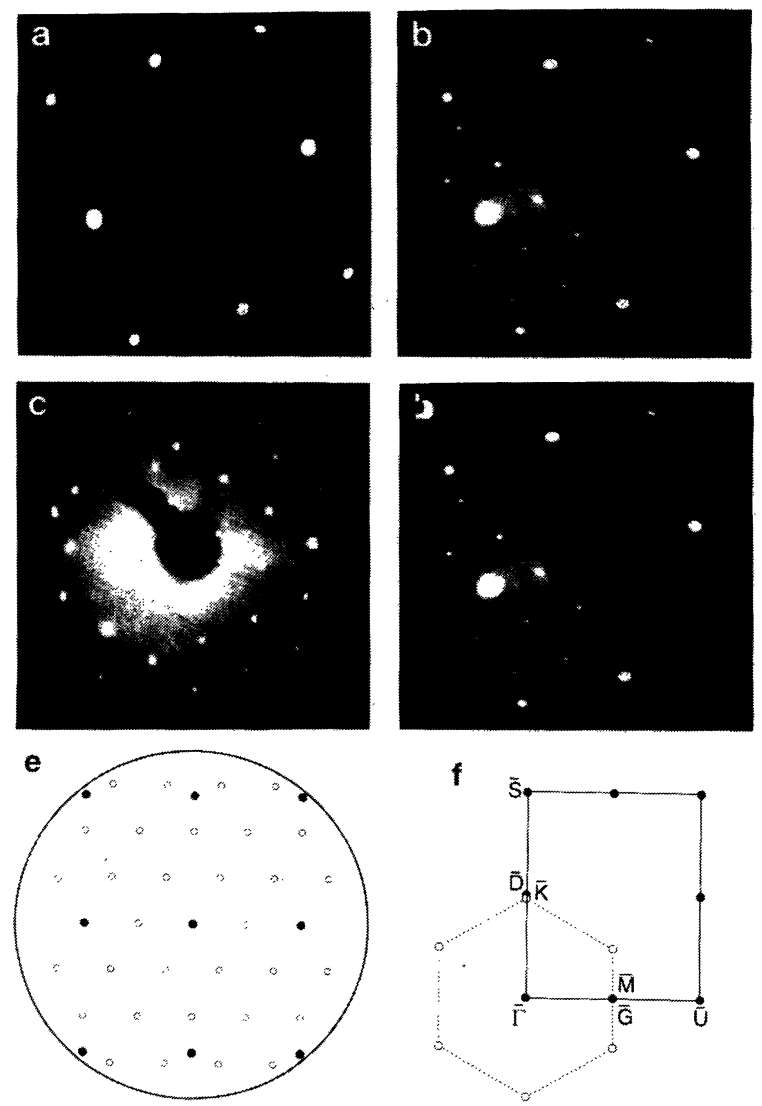

$\mathbf{f}$

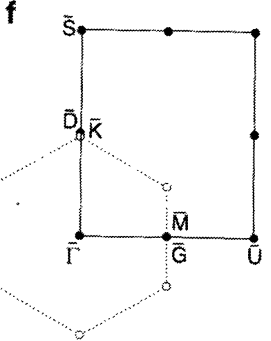

FIG. 1. LEED patterns recorded with $E_{p}=35 \mathrm{eV}$ of (a) a cleaved $\mathrm{GeS}(001)$ substrate; (b) $0.33 \mathrm{ML}$ and (c) $0.66 \mathrm{ML}$ of $\mathrm{C}_{60}$ on $\mathrm{GeS}$; both $\mathrm{C}_{60}$ and substrate spots are visible and they are identified by open and filled circles respectively in (e); (d) $1 \mathrm{ML}$ of $\mathrm{C}_{60}$ on $\mathrm{GeS}$; only spots of the fcc $\mathrm{C}_{60}(111)$ plane are visible; (f) orientations of the first surface Brillouin zone of $\mathrm{C}_{60}(111)$ and the $\mathrm{GeS}(001)$ substrate: the $\bar{\Gamma}-\bar{K}(\bar{\Gamma}-\bar{M})$ direction of the $\mathrm{C}_{60}$ SBZ is parallel to the $\bar{\Gamma}-\bar{D}-\bar{S}(\bar{\Gamma}-\bar{G}-\bar{U})$ direction of the substrate SBZ. 
the $\mathrm{C}_{60}$ hexagonal arrangement corresponding to the (111) plane of the room-temperature fcc phase are of very good quality, with sharp spots devoid of fine structure and a rather low background. For coverages lower than $1 \mathrm{ML}$, spots characteristic of both $\mathrm{C}_{60}(111)$ and $\mathrm{GeS}(001)$ are visible, the larger rectangular mesh [identified by dots in Fig. 1(e)] originating from the substrate. A weak but very sharp diffraction pattern characteristic of the (111) plane of $\mathrm{C}_{60}$ is already observed after an evaporation time of $45 \mathrm{~s}$, e.g., at the very low coverage of $0.04 \mathrm{ML}$. This together with the continuous decrease in intensity of the substrate spots with increasing coverage and the complete disappearance for coverages higher than $1 \mathrm{ML}$ might suggest a layer-by-layer growth mode.

The simultaneous observation at low coverages of the substrate and adsorbate spots allows one to specify the geometry of the epitaxy. Indeed, from inspection of Figs. 1 (b) and 1 (c) it is evident that in the reciprocal space the $\bar{\Gamma}-\bar{K}(\bar{\Gamma}-\bar{M})$ direction of the first $\mathrm{C}_{60}(111)$ surface Brillouin zone (SBZ) is aligned with the $\bar{\Gamma}-\bar{D}-\bar{S}(\bar{\Gamma}-\bar{G}-\bar{U})$ direction of the $\mathrm{GeS}(001) \mathrm{SBZ}$ as sketched in Fig. 1(f). In the direct space this means that the $\mathrm{C}_{60}(111)$ hexagonal layer is aligned with the substrate so that the $C_{60}$ [101] direction is parallel to the GeS $b$ axis and the $\mathrm{C}_{60}[1 \overline{2} 1]$ direction is parallel to the GeS $a$ axis (Fig. 2).

\section{B. TEM}

The interface geometry deduced from the LEED analyses is confirmed by SAED of a 200 - $\AA$-thick $\mathrm{C}_{60}$ film on $\mathrm{GeS}(001)$. Figure 3(a) shows the diffraction pattern obtained along the plane-view direction, i.e., along the substrate normal. Since the electrons enter the substrate first, each electron beam diffracted by the $\mathrm{GeS}(001)$ lattice acts as an incoming electron beam for the $\mathrm{C}_{60}(111)$ lattice. The quite complicated diffraction pattern of Fig. 3(a) can therefore be reconstructed by superposing on

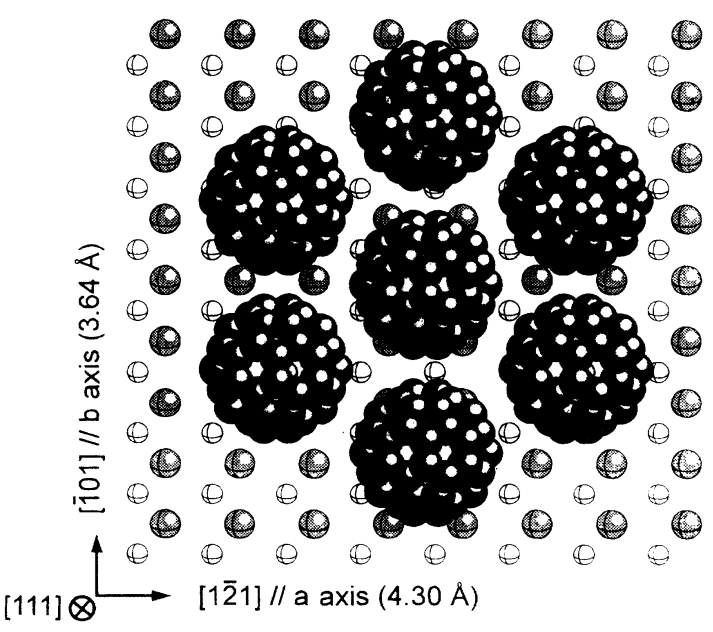

FIG. 2. Top view of the model structure of a $\mathrm{C}_{60}$ (111) layer grown on a $\mathrm{GeS}(001)$ substrate. Small grey (white) spheres represent the surface germanium (sulfur) atoms of $\mathrm{GeS}(001)$. The $\mathrm{C}_{60}[\overline{101}]([1 \overline{2} 1])$ direction is parallel to the $\mathrm{GeS} b$ axis $(a$ axis) so that the $\mathrm{C}_{60}(111)$ plane is parallel to the surface.
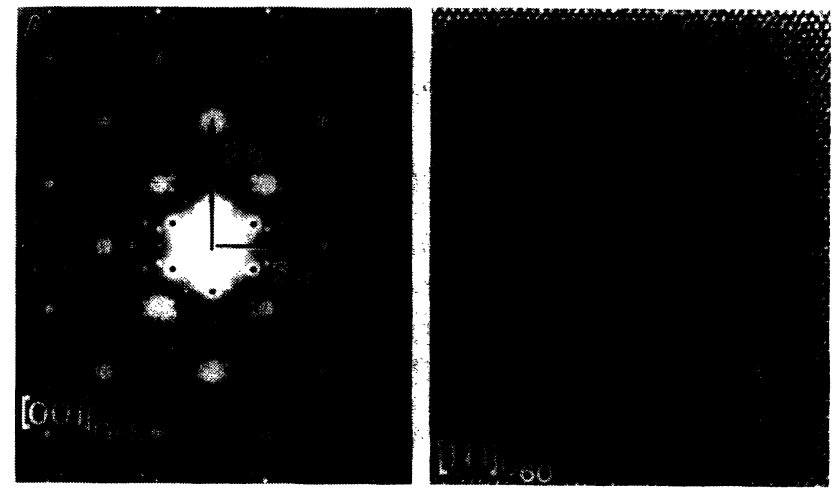

FIG. 3. (a) Selected-area diffraction pattern of a 200 - $\AA$-thick $\mathrm{C}_{60}$ film on $\mathrm{GeS}(001)$ along the [001] zone of the substrate. The reciprocal base vectors of the substrate are indicated; due to systematic extinctions the $I O O$ and $\mathrm{OIO}$ ( $I$ odd) reflections are not visible. A schematic explanation of the complete diffraction pattern is given in Fig. 4. (b) High-resolution image of a $\mathrm{C}_{60}$ film on $\mathrm{GeS}(001)$ viewed along the [111] zone. Note the perfect structure of the film continuing over large areas.

each spot of the $[001]^{*}$ diffraction pattern of GeS [Fig. 4(a)] a complete $\mathrm{C}_{60}[111]^{*}$ diffraction pattern [Fig. 4(b)]. Assuming that the $\mathrm{C}_{60}[1 \overline{2} 1]$ direction is parallel to the GeS[100] direction as shown in Fig. 4(b) yields then the complete diffraction pattern shown in Fig. 4(c). The calculated diffraction pattern of Fig. 4(c) reproduces exactly the most intense diffraction structures of Fig. 3(a). Additional weaker reflections in the diffraction pattern of Fig. 3(a) are due to higher-order reflections or double diffraction. The perfect agreement between the diffraction pattern of Fig. 3(a) and the calculated one of Fig. 4(c) allows one to deduce the following orientational relation between the film and the substrate (see Fig. 4):

$$
\begin{aligned}
& C_{60}[111] \text { is parallel to } \mathrm{GeS}[100], \\
& \mathrm{C}_{60}[\overline{101}] \text { is parallel to } \mathrm{GeS}[010], \\
& \mathrm{C}_{60}[\overline{1} \overline{1} 1] \text { is parallel to } \mathrm{GeS}[100],
\end{aligned}
$$

in agreement with the LEED analyses. Note that a slight misalignment between these directions of even less than $1^{\circ}$ would be immediately apparent as a distortion of the diffraction structures, e.g., the one outlined in Fig. 4(c). This is the case, for example, for the growth of $\mathrm{C}_{70} / \mathrm{GeS}$ where a misalignment of approximately $2^{\circ}$ between the [101] direction and the substrate $b$ axis is observed. ${ }^{41}$ The high symmetry of this diffraction structure in the case of $\mathrm{C}_{60}$ tells us that the alignment is perfect.

The sequence of the layers parallel to the substrate cannot be revealed directly, but the diffraction patterns can give strong indications. All reflections visible on the [111 ${ }^{*}$ diffraction pattern of $\mathrm{C}_{60}$ have indices which are all odd or all even, characteristic for a fcc structure and an $\cdots a b c a b c \cdots$ stacking. This fcc structure was also confirmed by diffraction patterns along other zones. In most cases no extra reflections are observed indicating that the $\cdots a b c \cdots$ stacking parallel to the substrate surface is close to perfect, i.e., very few stacking faults parallel to the substrate surface are present. Only in 
some few regions are weak extra spots observed, pointing towards the occasional presence of stacking faults.

Figure 3(b) shows a high-resolution image of the $\mathrm{C}_{60}$ film on top of the GeS surface imaged along the [111] direction. The imaging conditions are such that only the structure of the film is visible. Channels in the $\mathrm{C}_{60}$ crystal structure correspond to the bright dots. The hexagonal arrangement of the molecules is clearly shown and grains without any visible defect were found to extend over areas beyond $1 \times 1 \mu \mathrm{m}^{2}$, indicating a very high crystallinity of the film and the absence of structural defects.

\section{Model structure of the interface}

The fact that the GeS substrate acts as such a perfect template for the growth of crystalline fullerite films stems from the close match (see Fig. 2) between twice the lattice parameter of GeS along its $a$ axis $(2 \times 4.30 \AA=8.6 \AA)$ and the separation between rows of $\mathrm{C}_{60}$ clusters along [1 $1 \overline{2} 1]$ or equivalent directions $(8.68 \AA)$. Furthermore, the $\mathrm{GeS}(001)$ surface exhibits a peculiar corrugation in its basal plane, which contributes to induce the epitaxy.

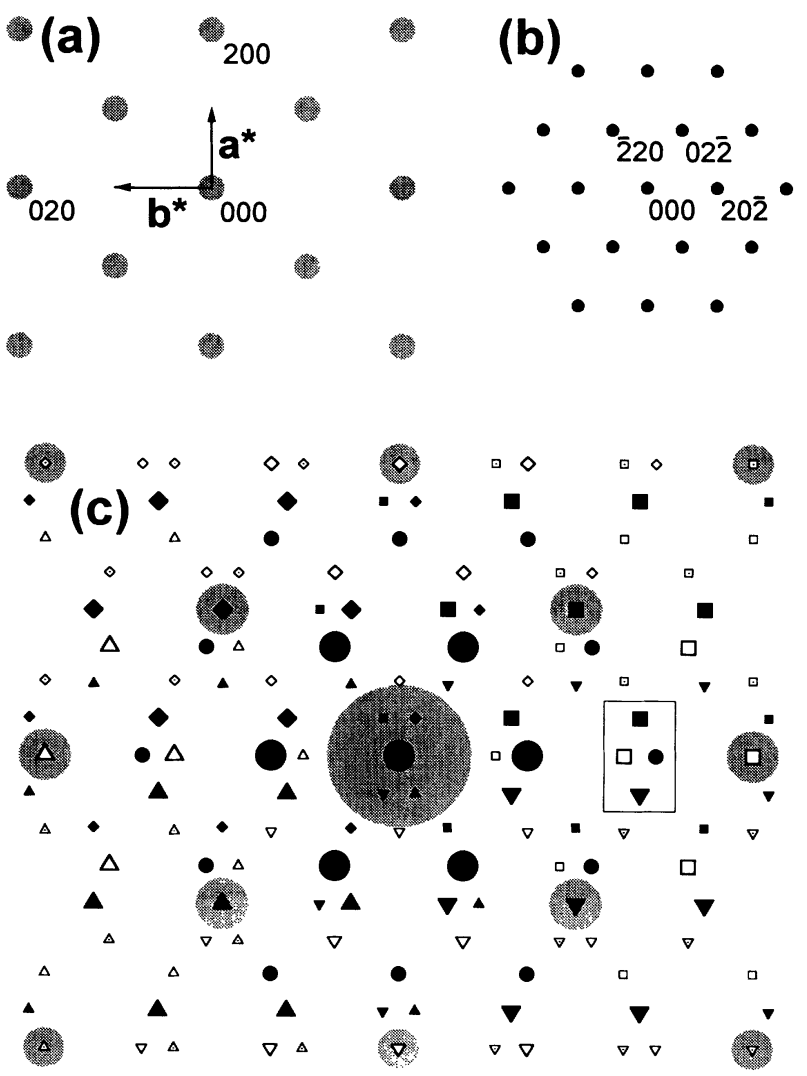

FIG. 4. Schematic diffraction patterns of (a) $\mathrm{GeS}(001)$ and (b) $\mathrm{C}_{60}$ (111). (c) Diffraction pattern of a thin $\mathrm{C}_{60}(111)$ film on $\mathrm{GeS}(001)$ obtained by superposing on each GeS diffraction spot a complete $\mathrm{C}_{60}[111]^{*}$ diffraction pattern. The different $\mathbf{C}_{60}$ $[111]^{*}$ diffraction patterns associated with each GeS spot are represented by different symbols. The size of the symbols decreases with increasing diffraction order so as to reproduce more or less the experimental intensities of the diffraction spots of Fig. 3(a).
This is sketched in Fig. 5, which presents a side view of the model structure. As a matter of fact, in a single layer of the orthorhombic structure of the monochalcogenide, each atom is covalently bonded to three nearest neighbors, forming zigzag chains along the $b$ axis with alternating positions perpendicular to the basal plane. Consequently, the cleaved surface presents the aspect of a corrugated roof due to the alignment of well-separated Ge-S zigzag chains at the selvedge, which provide a preferential adsorption direction in the interchain grooves along the $b$ axis. These one-dimensional corrugations with a periodicity of $4.3 \AA$ allow the easy alignment of $C_{60}$ [1̄01] rows along the substrate $b$ axis. The completion of the first monolayer is accomplished, at the expenses of a very small lattice distortion, if the $\mathrm{C}_{60}$ rows skip one interchain groove systematically, thereby allowing nearperfect arrangement at the interface. Notice that perfect registry of the first layer must be restricted to the $a$ direction, since the corrugation along the $b$ axis is much smoother, and there is no commensurability in this direction.

\section{GROWTH-MODE STUDY}

\section{A. Analysis of the XPS intensities}

In order to characterize the growth mechanism and determine the surface coverage of vapor-deposited $\mathrm{C}_{60}$ on $\mathrm{GeS}(001)$ we have monitored the XPS emission from core levels of the substrate $(\mathrm{Ge} 3 d)$ and the adsorbate $(\mathrm{C} 1 s)$ as a function of $\mathrm{C}_{60}$ deposition time. Using the intensity ratio of the $\mathrm{C} 1 s\left(I_{a}\right)$ and $\mathrm{Ge} 3 d\left(I_{s}\right)$ lines one can calculate the thickness $d$ of the $\mathrm{C}_{60}$ film using the relation

$$
d=\lambda \ln \left(1+\frac{I_{s}^{0}}{I_{a}^{\infty}} \frac{I_{a}}{I_{s}}\right),
$$

where $I_{s}^{0}$ is the Ge $3 d$ peak intensity of the bare substrate, $I_{a}^{\infty}$ is the C $1 s$ peak intensity of a thick $\mathrm{C}_{60}$ film for which the substrate emission features are completely attenuated, and $\lambda$ is the electron mean free path in $\mathrm{C}_{60}$. Independently of the value of $\lambda$, the plot of the thickness versus the deposition time presents a "break" at $18 \mathrm{~min}$, which indicates a change in sticking coefficient between the first monolayer and the following ones. Based on a lattice parameter of $14.17 \AA$ for fcc fullerite, the spacing between the (111) planes is $8.18 \AA$. Assuming that one has reached a coverage of $1 \mathrm{ML}$, i.e., a thickness of $8.18 \AA$

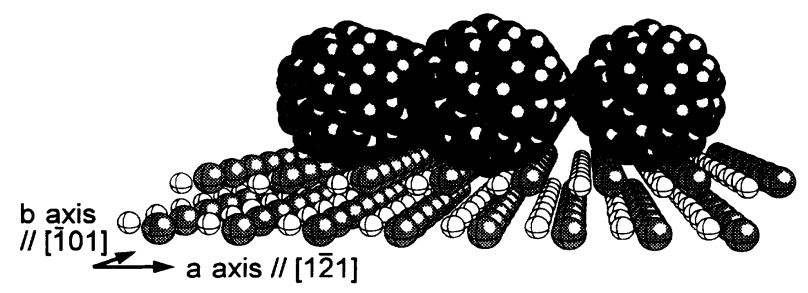

FIG. 5. Side view of the model structure of $a \mathrm{C}_{60}(111)$ monolayer on a $\mathrm{GeS}(001)$ substrate showing the peculiar corrugation in the $a$ direction of the basal plane and the systematic skipping of one interchain groove by the big $\mathrm{C}_{60}$ molecule. 
after $18 \mathrm{~min}\left(t_{1}\right)$ of evaporation yields an electron mean free path of $\lambda=17 \AA$. Under these assumptions one obtains the plot of the thickness versus deposition time represented in Fig. 6. The error bars have been calculated assuming an uncertainty of $5 \%$ on the intensities and of $2.3 \%$ on the mean free path $\lambda$, which is equivalent to a precision of $0.2 \AA$ on the measurement of the monolayer thickness. The solid line is the result of a weighted least squares fit. According to this fit, a coverage of $2 \mathrm{ML}$, i.e., a thickness of $16.4 \AA$, is reached after $43 \mathrm{~min}$, yielding a time of $25 \mathrm{~min}\left(t_{2}\right)$ for the completion of the second monolayer. Assuming that the $\mathrm{C}_{60}$ flux was constant over the entire duration of the experiment, the ratio of $t_{2}$ and $t_{1}$ gives then a ratio of the sticking coefficients of the first to the second monolayer $\left(s_{1} / s_{2}\right)$ of 1.4 . Consequently, the adsorption energy of a $\mathrm{C}_{60}$ cluster on $\mathrm{GeS}(001)$ seems to be slightly higher than on fullerite.

Applying Eq. (1) for calculating the film thickness we assumed implicitly a layer-by-layer or so-called Franck-Van der Merwe (FM) growth mode. We have therefore to check whether the evolution of the substrate and adsorbate core-level line intensities with coverage is consistent with this assumption. If both curves can be fitted with the same parameters (within some experimental error bar) as the one obtained from the thickness plot in Fig. 6 the procedure can be judged to be self-consistent and we can decide on a layer-by-layer growth mechanism.

Figure 7 shows the intensity plot of the Ge $3 d$ corelevel peak versus the coverage and deposition time. The coverage scale is not linear due to the difference in sticking coefficient between the first and the following monolayers. The shape of the curve seems to be consistent with a FM growth mode. Indeed, the experimental intensities (normalized to the one of the bare substrate) can be

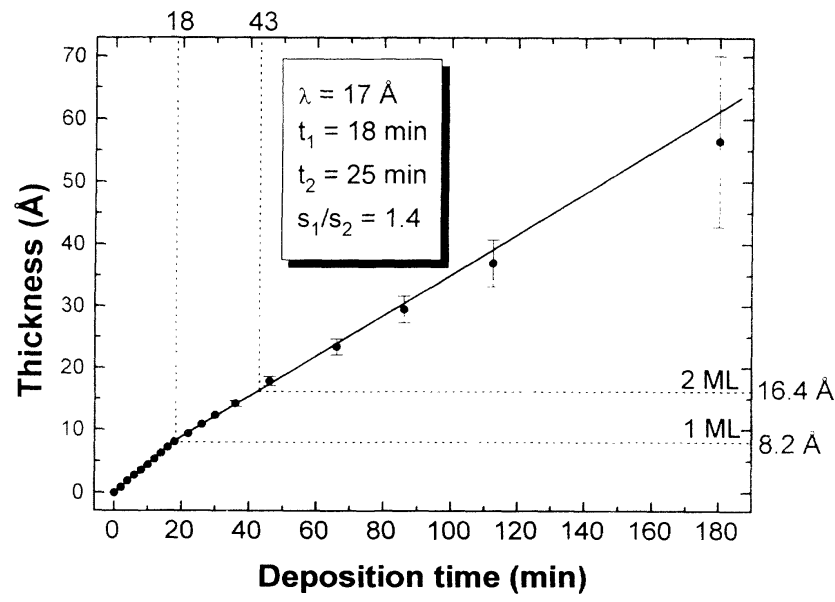

FIG. 6. Thickness of the vapor-deposited $\mathrm{C}_{60}$ layer versus deposition time calculated using the intensity ratio of the $\mathrm{C} 1 \mathrm{~s}$ and $\mathrm{Ge} 3 d$ core-level lines. The change in slope at $18 \mathrm{~min}$ corresponds to the completion of the first monolayer and indicates a difference in sticking coefficient between the first $\left(s_{1}\right)$ and the subsequent monolayers $\left(s_{2}\right) . t_{1}$ and $t_{2}$ correspond, respectively, to the time necessary to complete the first and the second monolayer. The mean free path $\lambda$ has to be taken equal to $17 \AA$ in order to yield a thickness of $8.2 \AA$ ( $1 \mathrm{ML}$ ) after $18 \mathrm{~min}$.

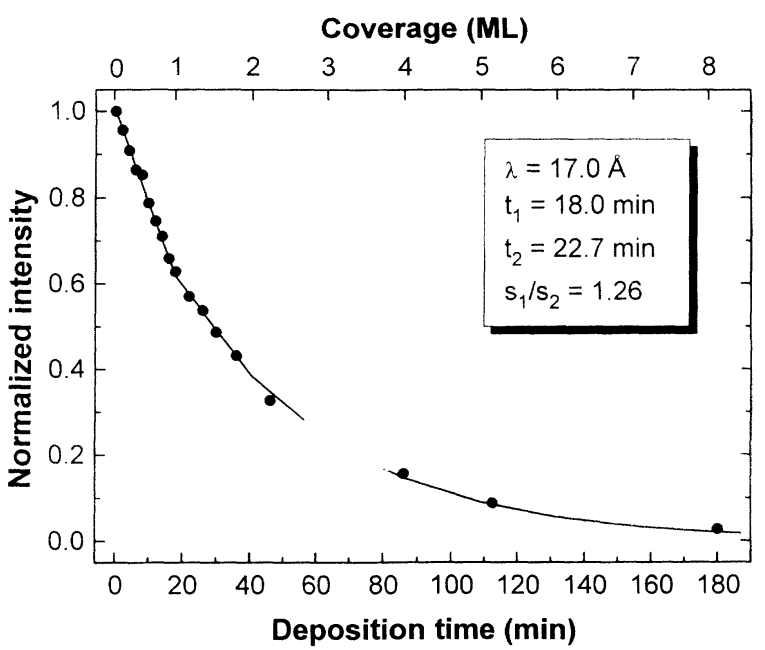

FIG. 7. Ge $3 d$ core-level line intensity versus deposition time and coverage. The solid line is a least-squares fit assuming a layer-by-layer growth mode. The values of the fitting parameters are listed in the inset.

very well fitted to a succession of linear segments ${ }^{42}$ given by the equation

$I_{s}=(n+1-\theta) e^{-n d / \lambda}+(\theta-n) e^{-(n+1) d / \lambda}$,

where $\theta$ is the coverage, $n$ is the number of already completed monolayers, i.e., $n=\operatorname{int}(\theta)$, and $d$ is the thickness of $1 \mathrm{ML}(8.18 \AA)$. As parameters for the fit we used the electron mean free path $(\lambda)$ and the time $\left(t_{2}\right)$ to grow one $\mathrm{C}_{60}$ monolayer on fullerite. The time to complete the first monolayer $\left(t_{1}\right)$ was kept constant and equal to the value obtained from Fig. 6 (18 min). The results of the fit are $\lambda=17 \AA$ and $t_{2}=22.7 \mathrm{~min}$, in good agreement with the values obtained from the fit of the thickness plot in Fig. 6.

The C $1 s$ core-level intensity as a function of the coverage is shown in Fig. 8. The evolution of the adsorbate intensity $I_{a}$ (normalized to the one of bulk $\mathrm{C}_{60}$ ) in the case

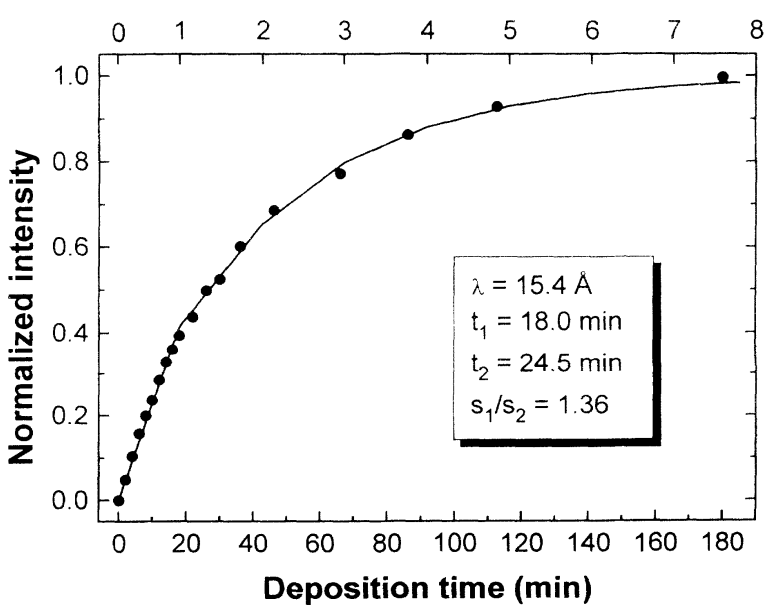

FIG. 8. C $1 s$ core-level line intensity versus deposition time and coverage. The solid line is a least-squares fit assuming a layer-by-layer growth mode. The values of the fitting parameters are listed in the inset. 
of a FM growth mode follows the equation

$$
I_{a}=1-I_{s},
$$

where $I_{s}$ is given by (2). Again, with optimized parameter values of $\lambda=15.4 \AA$ and $t_{2}=24.5 \mathrm{~min}$ the experimental curve is nearly perfectly fitted. The mean free path of the $\mathrm{C} 1 \mathrm{~s}$ photoelectron is found to be slightly smaller than for the Ge $3 d$ photoelectron. This is consistent with the "universal curve" as the kinetic energy of the Ge $3 d$ photoelectron is $\sim 250 \mathrm{eV}$ higher than that of the $\mathrm{C} 1 \mathrm{~s}$ one $(\sim 1200 \mathrm{eV})$.

In conclusion, the analysis of the behavior of the corelevel intensities of the substrate and the adsorbate proves that $\mathrm{C}_{60}$ grows in a layer-by-layer mode on the lamellar substrate $\mathrm{GeS}(001)$. It gives the following estimates for the mean free path $\lambda$ and the sticking coefficient ratio $s_{1} / s_{2}$ of the first and second monolayers:

$$
\begin{aligned}
& 15.4<\lambda<17.0 \AA, \\
& 1.26<\frac{s_{1}}{s_{2}}<1.4 .
\end{aligned}
$$

The small difference in adsorption energy between the first and second monolayers raises questions about the first-layer bonding which will be addressed later on.

\section{B. Reflection $x$-ray scattering}

The layer-by-layer growth is further confirmed by synchrotron reflection x-ray scattering (RXS) measurements. The preparation of the sample used for this experiment differs slightly from the one described above because the experimental setup of the Seya-Namioka beamline did not allow heating of the substrate during the evaporation. The films were therefore grown in several steps. We sublimed about $1 \mathrm{ML}$ of $\mathrm{C}_{60}$ and annealed the sample at about $180^{\circ} \mathrm{C}$ in order to obtain an epitaxial monolayer as evidenced by the sharp hexagonal LEED pattern. The evaporation and annealing cycle was then repeated several times for longer evaporation periods (each evaporation corresponding to about $4 \mathrm{ML}$ ) until the desired film thickness was obtained. After each cycle the quality of the film was checked by LEED. Judging from the sharpness of the LEED spots the quality of the films grown in this way is comparable to that obtained by the conventional method described above. Films which were not annealed after evaporation also exhibit LEED patterns but the sharpness of the diffraction spots is greatly improved by annealing the sample.

$\theta-2 \theta$ "Bragg" scans were recorded in the RXS geometry, i.e., along the specular direction of the $\mathrm{GeS}(001)$ substrate, which allows one to probe diffraction planes parallel to the substrate. Figure 9 shows a part of a RXS Bragg scan from a 150-Å-thick film on GeS(001). $Q$ is the scattering vector component normal to the sample surface. Only the $\{00 L\}$ family of the GeS peaks and $\{H H H\}$ family of $\mathrm{C}_{60}$ peaks are seen in such a scan, confirming once more that the $\mathrm{C}_{60}\{111\}$ planes are parallel to the substrate. The sharp peak at $0.603 \AA^{-1}$ corresponds to the (001) reflection of the substrate. This yields

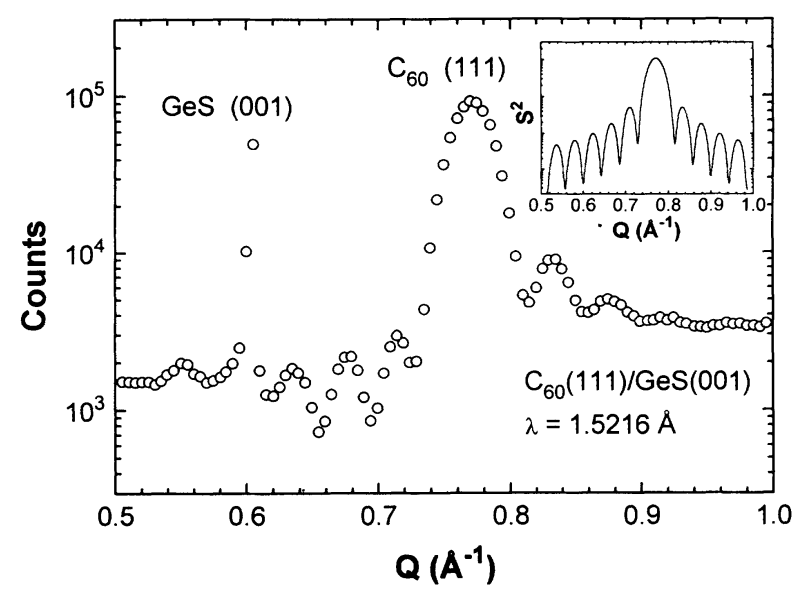

FIG. 9. $\theta-2 \theta$ Bragg scan recorded in the reflection geometry of the (001) GeS peak and the (111) $\mathrm{C}_{60}$ peak. The abscissa is the amplitude of the scattering vector $Q=4 \pi \sin \theta / \lambda$. Finitesize oscillations are observed in the (111) Bragg reflection indicating layer-by-layer growth. The inset shows the calculated structure factor $S^{2}$ for $18 \mathrm{C}_{60}$ layers with an interlayer distance of $8.15 \AA$.

a lattice parameter of $c=10.42 \pm 0.02 \AA^{-1}$ in good agreement with the value reported by Grandke and Ley (10.44 $\AA)^{43}$ A clear peak corresponding to the (111) $\mathrm{C}_{60}$ Bragg reflection is observed at $0.771 \AA^{-1}$ together with finitesize oscillations, which arise from the interference of the beams diffracted by the limited number of (111) planes. Indeed, the structure factor $S$ of a film made up of $N$ layers with interlayer distance $d$ is given by the well-known equation

$$
S^{2}=\frac{\sin ^{2}(N Q d / 2)}{\sin ^{2}(Q d / 2)},
$$

which is plotted in the inset of Fig. 9. The nice correspondence between the maxima in the structure factor and the finite-size oscillations observed in the experimental spectra is obvious. The observation of such clear and well-defined finite-size oscillations requires nearly perfectly flat surfaces with a large domain size, as they are obtained only in the case of a layer-by-layer growth mode. If one is in the presence of a three-dimensional island growth mechanism the diffuse scattering induced by the surface roughness wipes out the weak finite-size oscillations, resulting simply in the observation of a broadened diffraction peak. The observation of finite-size oscillations on the (111) Bragg reflection confirms therefore the layer-by-layer growth and points to the high crystalline quality of the sample. The peak position of $0.771 \AA^{-1}$ of the (111) reflection gives an interlayer distance of $d=8.15 \pm 0.02 \AA$, close to the value for bulk $\mathrm{C}_{60}$ $(8.18 \AA),{ }^{44}$ showing that the distortion induced by the substrate strain is small within the experimental precision. From the width of the $\mathrm{C}_{60}(111)$ Bragg peak, the number of layers can be estimated to be $N=q_{(111)} / \Delta q \approx 18$, where $q_{(111)}=0.771 \AA^{-1}$ and $\Delta q=0.043 \AA^{-1}$ is the distance between the peak maximum $\left(q_{(111)}\right)$ and the first minimum. 


\section{ELECTRONIC STRUCTURE OF THE INTERFACE}

\section{A. Core levels and shake-up}

In the gas and solid phases, all carbon atom sites of a $\mathrm{C}_{60}$ molecule are equivalent. Indeed, a comparison between the XPS C $1 s$ line of a thick $\mathrm{C}_{60}$ film and that of highly oriented pyrolytic graphite (HOPG) graphite (for which all the carbon sites are also equivalent) shows that they have nearly the same line shape on the low-bindingenergy side [Fig. 10(a)]. The line shapes on the highbinding-energy side are different because they are determined by the electron-hole, phonon, and plasmon excitations, which are of course different for $\mathrm{C}_{60}$ and graphite.

Upon adsorption on a solid surface the different carbon sites on a $\mathrm{C}_{60}$ molecule will become inequivalent due to the interaction with the substrate. In the case of chemisorption new bonding configurations will be formed, which will affect differently the carbon atoms on the interface side and the ones on the opposite pole of the molecule. If the $\mathrm{C}_{60}$-substrate interaction is of the van der Waals type (physisorption) the interaction of the fluctuating dipoles with their image charges, as the molecule approaches the surface, will induce a net positive dipole ${ }^{45}$ across the molecule and give rise to slight inequivalencies of the carbon sites on the molecule. While for chem-

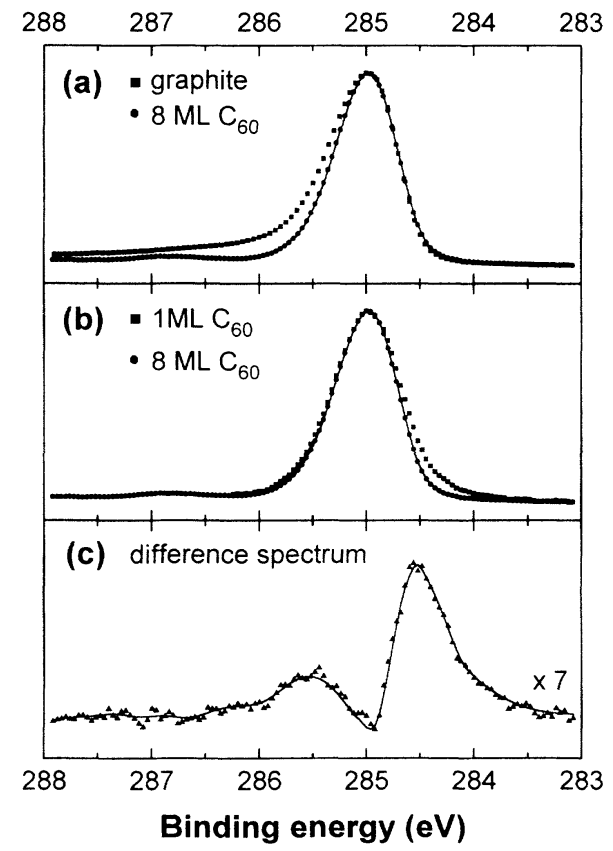

FIG. 10. (a) Comparison between the XPS C $1 s$ line of graph-

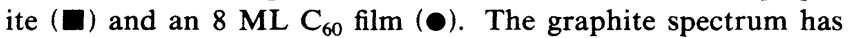
been shifted down by $0.6 \mathrm{eV}$ to higher binding energies. The shapes of the low-binding-energy sides of both peaks are nearly identical, indicating that in a $\mathrm{C}_{60}$ molecule all the carbon atoms are equivalent, as in graphite. (b) The XPS C $1 s$ line at $285.0 \mathrm{eV}$ of an $8 \mathrm{ML}(\bullet)$ and a $1 \mathrm{ML}(\boldsymbol{\square}) \mathrm{C}_{60}$ film shows a pronounced line-shape change on the low-binding-energy side compared to the one of an $8 \mathrm{ML} \mathrm{C}_{60}$ film. (c) The difference spectrum ( $\Delta$ ) of the two $\mathrm{C} 1 s$ lines shows a peak at about $284.5 \mathrm{eV}$ which indicates charge transfer to the carbon atoms at the interface. isorption the $\mathrm{C}$ is peak should show a large shift and broadening (or even splitting up into several components), ${ }^{1,24,26}$ as well as a strong dependence on the coverage, physisorption should result only in a weak dependence of the $\mathrm{C} 1 s$ line position and line shape on the coverage. $^{24}$

In Fig. 10(b) we compare the C $1 s$ signal of a $1 \mathrm{ML}$ film on $\mathrm{GeS}$ to the $8 \mathrm{ML}$ film one, which is taken as a reference for bulk $\mathrm{C}_{60}$. While the $1 \mathrm{ML}$ sample $\mathrm{C} 1 \mathrm{~s}$ peak does not shift in energy with respect to the thick-film one, indicating that the interaction is mainly of the van der Waals type, one observes a small but significant difference in the spectra line shapes: the $1 \mathrm{ML}$ one has a larger tail on the low-binding-energy side. If one normalizes the two spectra to the same intensity and calculates the difference, one obtains the curve plotted in Fig. 10(c), which shows a clear peak at $284.5 \mathrm{eV}$. We suggest that this peak is the contribution from the carbon atoms located at the GeS interface which, due their different chemical environment, have a slightly different binding from that of the carbon atoms on the top of the molecule. This peak area represents 8-9\% of the total $1 \mathrm{ML} \mathrm{C} 1$ s signal, which corresponds to five or six carbon atoms. This might indicate that beyond the van der Waals interaction there could be an additional interaction mediated by a five- or six-membered ring of the molecule, such as, for example, a small charge delocalization.

To ascertain that the contribution at $284.5 \mathrm{eV}$ is due to carbon atoms in direct "contact" with the GeS substrate, we have followed the $\mathrm{C} 1 s$ line-shape evolution for higher coverages. We found that with increasing coverage the shape of the difference spectrum remains unchanged but its intensity decreases for coverages higher than $1 \mathrm{ML}$. The area of the $284.5 \mathrm{eV}$ peak as a function of the coverage follows the curve shown in Fig. 11. One observes a constant intensity up to $1 \mathrm{ML}$ followed by an exponential decrease for higher coverages. This behavior definitely demonstrates that only interface carbon atoms contribute

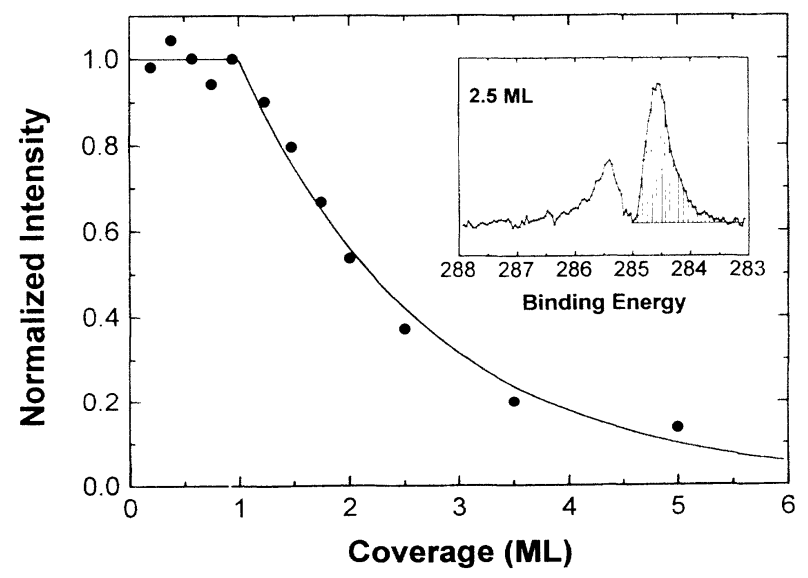

FIG. 11. Plot of the intensity of the low-binding-energy peak at $284.5 \mathrm{eV}$ in the difference spectrum (shown in the inset) versus $\mathrm{C}_{60}$ coverage. The intensity is constant up to $1 \mathrm{ML}$ and decreases then exponentially with increasing coverage, proving that the peak in the difference spectrum has to be attributed exclusively to the contribution of the interface carbon atoms. 
to the peak at $284.5 \mathrm{eV}$ in the difference spectrum. Indeed, for coverages lower than $1 \mathrm{ML}$ and for normal emission, the $\mathrm{C} 1 s$ signal from the interface carbon atoms is only attenuated by the molecule itself and thus independent of the coverage, while for higher coverages the signal is attenuated by the uppermost $\mathrm{C}_{60}$ layers and therefore decreases exponentially with increasing coverage.

At first sight, the shift to lower binding energies for the interface carbon atoms might indicate a small charge transfer from the substrate to the $\mathrm{C}_{60}$ molecules, similar to the one observed for $\mathrm{C}_{60}$ on metal substrates. $1,4,7,24-29$ However, as we will see in the next section, this interpretation is in contradiction with the observed downward bending of the substrate bands observed in the UPS measurements. We propose, therefore, that the observed shift to lower binding energies for the interface carbon atoms results from the more efficient screening, in particular image-charge screening, of the carbon atoms closest to the surface. In fact, due to the large size of the $\mathrm{C}_{60}$ molecule, the image potential decreases drastically over the diameter of a molecule.

A further argument for the inequivalence between the interface carbon atoms and the other atoms of the molecule is obtained by comparing the $\mathrm{C} 1 s$ core-level lines for normal emission and grazing emission $\left(5^{\circ}\right)$, as shown in Fig. 12. In the grazing-emission geometry,- electrons originating from the interface have to travel a distance $d=7.1 / \sin 5^{\circ}=80 \AA$ (remembering that the diameter of the molecule is $7.1 \AA$ ) before escaping from the film. With a mean free path of about $17 \AA$ it is then clear that only electrons ejected from the topmost carbon atoms will reach the analyzer. In contrast, in the normal emission spectrum, all the carbon atoms of the molecule will

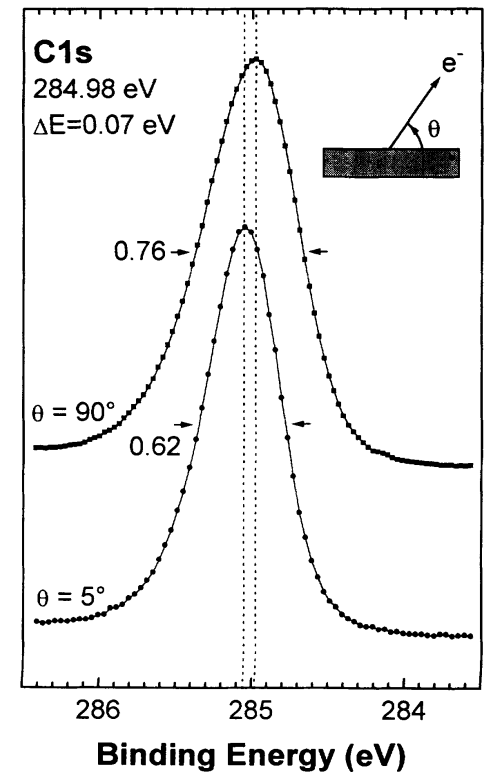

FIG. 12. C $1 s$ line shape for normal emission $\left(\theta=90^{\circ}\right)$ and for grazing incidence $\left(\theta=5^{\circ}\right)$. The grazing-emission spectrum is shifted to higher binding energies and has a smaller full width at half maximum $(0.62 \mathrm{eV}$ instead of $0.76 \mathrm{eV})$. contribute to the $\mathrm{C} 1 \mathrm{~s}$ signal. The comparison of the two spectra (Fig. 12) shows a $0.14 \mathrm{eV}$ broadening and a 0.07 $\mathrm{eV}$ shift to lower binding energy for the normal emission spectrum. If the angular-dependent experiment is repeated on a thick film, two identical line shapes and widths are observed but shifted by about $0.07 \mathrm{eV}$, the same amount as for the monolayer film. The higher binding energy of the topmost carbon atoms might be interpreted as a less efficient screening by the surrounding atoms (or molecules) of the photohole created in the topmost carbon atoms, as a result of the lower coordination at the surface.

In Fig. 13 we have reported the evolution of the $\mathrm{C} 1 \mathrm{~s}$ line position from submonolayer coverages up to $2 \mathrm{ML}$. The solid line in Fig. 13 should only be used as a guide for the eye and is not based on a physical model. Although the overall energy shift between the very low coverage and the bulk spectrum is small $(0.1 \mathrm{eV})$, though well within the experimental accuracy, one can clearly recognize that, starting from a very low coverage of 0.02 ML the C $1 s$ line position shifts very rapidly to higher energies with increasing coverage and reaches a value very close to the bulk value $(284.98 \mathrm{eV})$ before the completion of the first monolayer. The steep slope at low coverage might indicate that $\mathrm{C}_{60}$ adsorbs first near to defects or steps. Indeed, at a step the substrate-induced screening (image-charge screening and possibly charge-transfer screening) is more efficient than on a flat terrace, ${ }^{46,47}$ so that the binding energy is lowered. Adsorption at steps and defects in the initial stages of the $\mathrm{C}_{60}$ growth on $\mathrm{GeS}(001)$ has been confirmed by recent atomic-force microscopy (AFM) measurements ${ }^{48}$ and has been observed for $\mathrm{C}_{60}$ deposition on many other substrates. ${ }^{2,3,6,10,15}$ The fact that the $\mathrm{C} 1 \mathrm{~s}$ line position does not shift significantly for coverages higher than $0.5 \mathrm{ML}$ indicates that the substrate image-charge screening is small compared to the polarization screening by the neighboring molecules.

Although the carbon sites on the $\mathrm{C}_{60}$ molecule become inequivalent upon adsorption, the overall electronic structure is not severely disturbed. This can be seen from Fig. 14, where we compare the $\mathrm{C} 1 s$ shake-up spectra recorded for $1 \mathrm{ML}$ and a thick film. Both spectra have

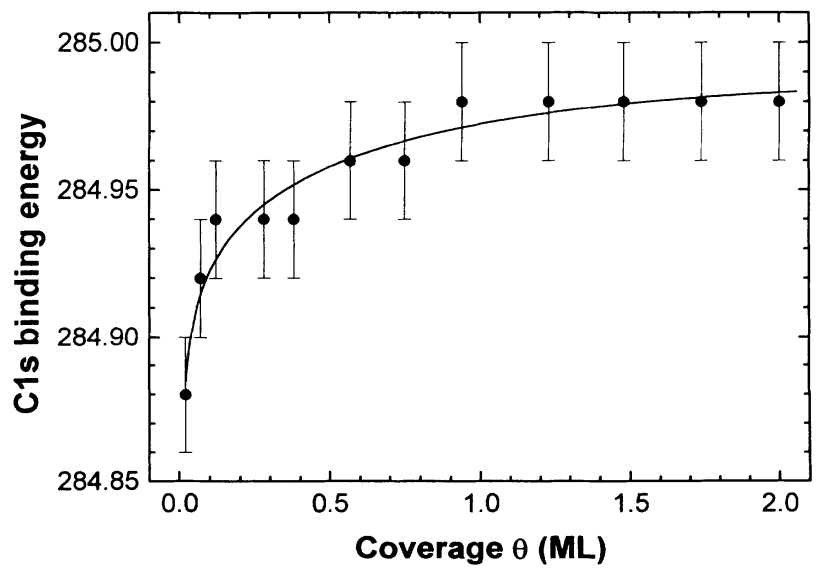

FIG. 13. Plot of the $\mathrm{C} 1 s$ line position versus coverage. 


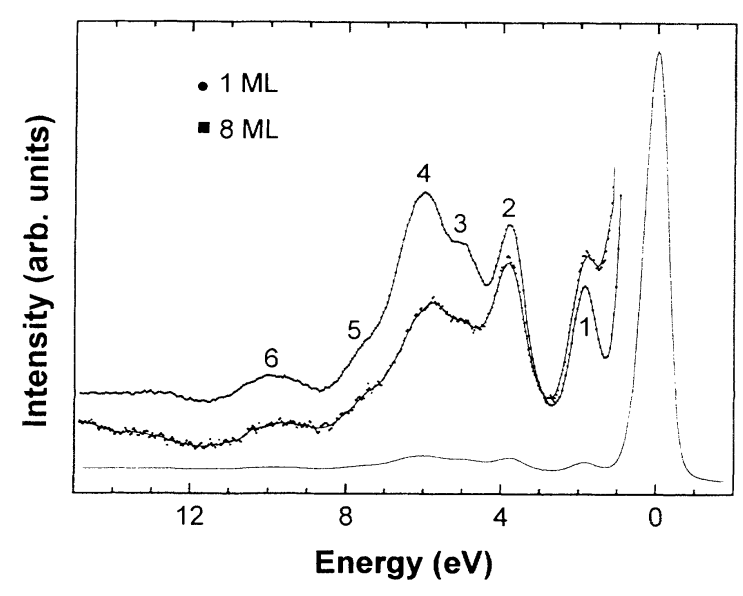

FIG. 14. Comparison between the $\mathrm{C} 1 s$ shake-up satellites of a monolayer and a thick $\mathrm{C}_{60}$ film. The energy is referenced with respect to the $\mathrm{C} 1 s$ main line. Each of the six well-defined satellites in the thick-film spectrum has a corresponding feature in the monolayer spectrum, but with lower intensity in the 4-12 $\mathrm{eV}$ range.

been normalized to the same C $1 s$ main line intensity. The six distinct shake-up structures observed in the thick-film sample are clearly recognized in the $1 \mathrm{ML}$ one, although slightly shifted and with lower intensity in the shake-up energy region of $4-12 \mathrm{eV}$. The sharpness of the shake-up features in the monolayer spectrum shows that the electronic structure of $\mathrm{C}_{60}$ is not perturbed by the GeS substrate: the hybridization between $\mathrm{C}_{60} p$ states and substrate $s$ and $p$ states is negligible. The higher intensity of the shake-up features for the thick-film sample can be explained by the contribution of the inelastic scattering, which should be strongly reduced in the case of a $\mathrm{C}_{60}$ monolayer. Indeed, if we subtract the $\mathrm{C} 1 s$ shake-up spectrum of the monolayer from the thick-film one, we obtain the spectrum plotted in Fig. 15. This difference spectrum closely resembles the electron-energy-loss

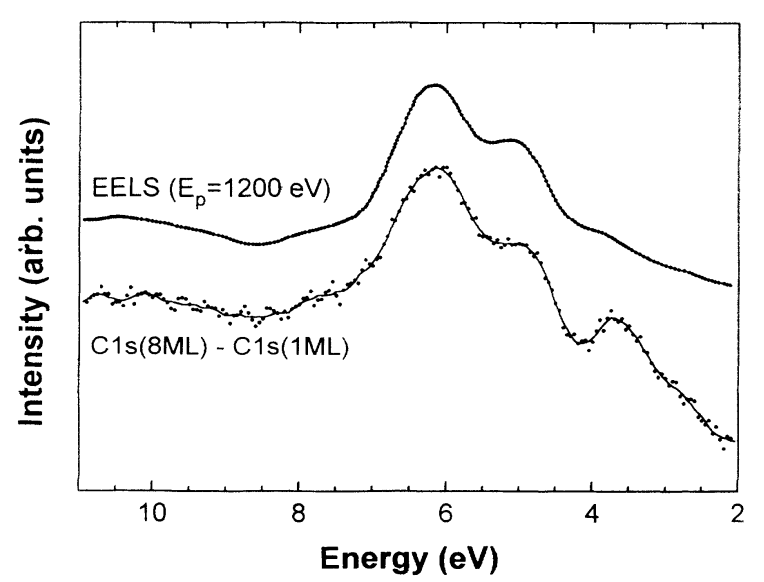

FIG. 15. Spectrum obtained by subtracting the $\mathrm{C} 1 \mathrm{~s}$ shake-up spectrum of a monolayer sample from the thick-film one and comparison with the inelastic losses suffered by electrons of kinetic energy close to that of a C $1 s$ photoelectron $(\sim 1200 \mathrm{eV})$. The EELS spectrum was taken from Ref. 49. fingerprint of a thick film recorded with a primary energy of $1200 \mathrm{eV},{ }^{49}$ an energy close to the kinetic energy of a C $1 s$ photoelectron. This definitely proves that the shakeup intensity difference (Fig. 14) is mainly due to the contribution of inelastic scattering and not to hybridization.

\section{B. Valence-band spectrum and substrate band bending}

More insight into the $\mathrm{C}_{60}$-substrate interaction, especially a possible small charge delocalization beyond the van der Waals interaction and the interface dipole, can be gained by following with UPS the evolution of the valence-band spectrum with increasing $\mathrm{C}_{60}$ coverage. Figure 16 shows a complete set of 14 valence-band spectra recorded for increasing $\mathrm{C}_{60}$ coverages ranging from 0 up to $8.1 \mathrm{ML}$.

According to pseudopotential energy-band calculations $^{50}$ the valence bands of the GeS substrate can be divided into three regions. ${ }^{51}$ The first region extending from 0 to $7 \mathrm{eV}$ binding energy, corresponding to features $a$ to $f$ in the $0 \mathrm{ML}$ spectrum of Fig. 16, is predominantly due to the bonding combination of the cation $\left(\mathrm{Ge} 4 s^{2} 4 p^{2}\right)$ and the anion $\left(\mathrm{S} 3 s^{2} 3 p^{4}\right) p$ electrons. Two other regions of bands at 7-10 eV (features $g$ and $h$ ) and 12-14 eV binding energies (not shown) are due to cation and anion $s$ electrons ( $s$ bands), respectively.

The 8.1 ML spectrum, taken as a reference for the bulk $\mathrm{C}_{60}$ valence band, shows well-defined features derived from the $\pi_{p}, \sigma_{p}$, and $\sigma_{s}$ levels. $^{52}$ The leading occupiedstate features, labeled 1 and 2, have $\pi_{u}$ and $\pi_{g}$ character with fivefold and ninefold degeneracy, respectively. Their radial charge densities are characterized by a node on the "spherical" shell formed by the atomic nuclei.

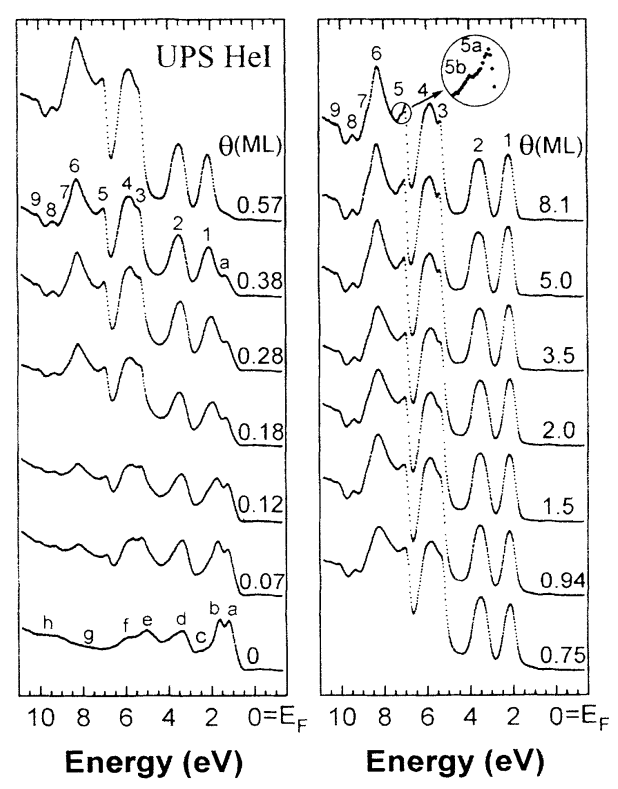

FIG. 16. Set of 14 UPS spectra excited by $\mathrm{He}$ I radiation $(h v=21.21 \mathrm{eV})$ for increasing $\mathrm{C}_{60}$ coverages. The GeS substrate features are labeled from $a$ to $h$, and the typical bulk $\mathrm{C}_{60}$ features are numbered from 1 to 9 . The binding energies are referenced with respect to the Fermi level. 
States 5-10 eV below $E_{F}$ are of mixed $\pi$ and $\sigma$ character, and those more than $\sim 10 \mathrm{eV}$ below $E_{F}$ are $\sigma$ derived.

The evolution of the valence-band spectrum with increasing coverage clearly shows that the molecular nature of the $\mathrm{C}_{60}$ molecule is preserved upon adsorption and that its electronic structure is only slightly perturbed. Indeed, most of the characteristic $\mathrm{C}_{60}$ features (labeled from 1 to 9) are discernible at the submonolayer coverages although slightly shifted and broadened with respect to the bulk reference spectrum $(8.1 \mathrm{ML})$. The sharpening of the molecular features with increasing coverage can be very nicely followed on feature 3 : it is seen as a shoulder on peak 4 for submonolayer coverages and is clearly resolved for coverages higher than $2 \mathrm{ML}$. In the same manner, two contributions ( $5 \mathrm{a}$ and $5 \mathrm{~b}$ ) are resolved in feature 5 for the bulk spectrum which are not discernible in the submonolayer spectra. The broadening of the molecular energy levels at low coverage is probably due to the symmetry reduction upon adsorption, which lifts the degeneracy of the levels and splits them up into several sublevels.

The weak features observed within about $1 \mathrm{eV}$ around the Fermi level are due to the $\mathrm{He}$ I satellite at $23.087 \mathrm{eV}$ and account for about $2 \%$ of the signal. A detailed analysis shows that these features can by no means be attributed to the formation of a new band resulting from a charge transfer from the substrate into a $\mathrm{C}_{60}$ band derived from the lowest unoccupied molecular orbital (LUMO). ${ }^{53-56}$ It appears then that there is no appreciable charge transfer from the substrate to the $\mathrm{C}_{60}$ molecule.

Because of the layer-by-layer growth and of the higher photoemission yield of $\mathrm{C}_{60}$ compared to $\mathrm{GeS}$, the intensity of the substrate peaks is strongly attenuated by the adsorbed molecules, and the photoemission features of $\mathrm{C}_{60}$ quickly dominate the valence-band spectrum as the coverage increases. At $0.38 \mathrm{ML}$, all the $\mathrm{C}_{60}$ features are clearly developed and the only detectable substrate feature is peak $a$, which is located within the fundamental $\mathrm{C}_{60}$ gap. Not being masked by the condensed $\mathrm{C}_{60}$ for coverages lower than $\sim 0.6 \mathrm{ML}$, it can be used as a monitor of the substrate condition. Figure 17 shows the valence band for coverages from 0 up to $0.75 \mathrm{ML}$ expanded in the region of the substrate peak $a$. One observes that the substrate peak shifts by an amount of about $0.2 \mathrm{eV}$ to higher binding energies with increasing coverage, indicating a downward substrate band bending or the formation of an electron accumulation layer at the interface. This observation rules out the charge delocalization of substrate electrons over the $\mathrm{C}_{60}$ molecule which has been reported for many $\mathrm{C}_{60} /$ metal interfaces, ${ }^{1,4,7,24-29}$ because this would imply an upward band bending. We are therefore forced to conclude that, on the contrary, electrons (or fractions of electrons) of the $\mathbf{C}_{60}$ molecule are delocalized over the substrate. This is plausible, because (1) as already mentioned, the van der Waals interaction of the $\mathrm{C}_{60}$ molecule with the substrate induces a positive dipole at the interface, ${ }^{45}$ i.e., a higher electron density at the interface; (2) GeS is slightly ionic, and the Ge cations are sticking out of the surface with respect to the $S$ anions, so that electrons on the $\mathrm{C}_{60}$ molecule may feel an additional

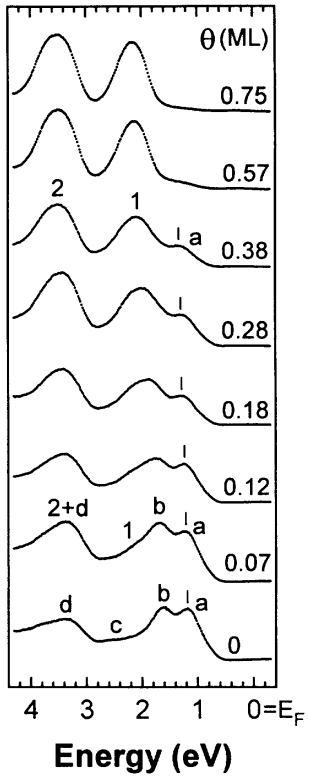

FIG. 17. UPS spectra of Fig. 16 expanded in the energy region $0-4 \mathrm{eV}$ and for coverages of 0 up to $0.75 \mathrm{ML}$. One can clearly follow the shift of the first GeS valence-band feature $a$ to higher binding energies with increasing $\mathrm{C}_{60}$ coverage.

attraction towards the substrate.

In order to compare in more detail the $\mathrm{C}_{60}$ valence band for low coverages (Fig. 16) to the bulk one we have subtracted the emission due to the GeS substrate. For each spectrum the GeS valence band was normalized to the residual intensity of peak $a$ and shifted in order to align the substrate features in both spectra before subtracting it from the corresponding spectrum of the $\mathrm{C}_{60^{-}}$ covered surface. The result is shown in Fig. 18 for two coverages: (a) $0.02 \mathrm{ML}$ (not shown in Fig. 16) and (b) 0.28 ML. The broadening and the shift of all the molecular levels towards the Fermi level relative to the thick-film
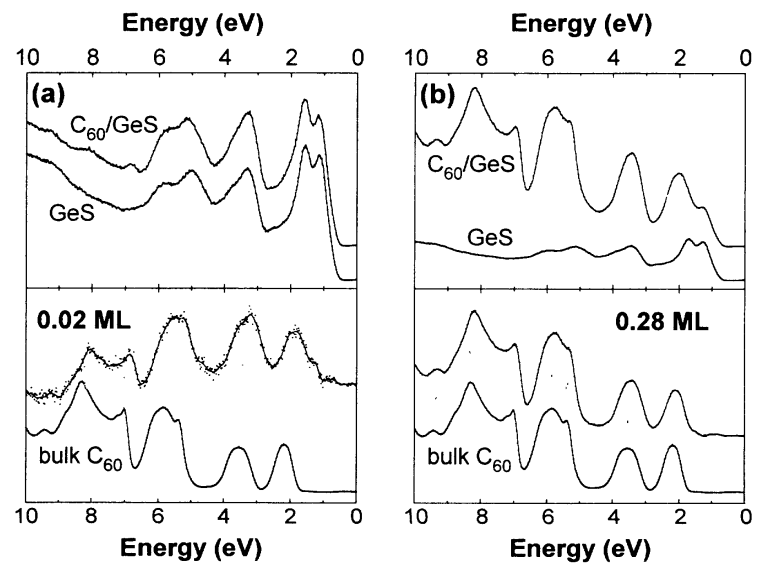

FIG. 18. Calculation of the difference spectrum (shown in the lower panels) of the UPS spectrum of the $\mathrm{C}_{60}$-covered surface and the shifted clean GeS spectrum (both shown in the upper panels) for two different coverages: (a) $0.02 \mathrm{ML}$ and (b) $0.28 \mathrm{ML}$. In the lower panel, the difference spectra are compared to the spectrum of bulk $\mathrm{C}_{60}$. 
spectrum is clearly visible, especially for the very low coverage spectrum. The shift is not exactly the same for all the molecular bands and decreases very rapidly with increasing coverage. This can be seen from Fig. 19, where we have plotted for each molecular feature and for the first substrate valence-band feature its binding energy versus coverage. The shift at low coverage is about 0.3 $\mathrm{eV}$ for the highest occupied molecular orbital (HOMO) (peak 1) and $0.2 \mathrm{eV}$ for the HOMO -1 . The stabilization of the shift long before the completion of the first monolayer confirms the adsorption near defects and terraces in the initial stage of the growth, already suggested by the XPS analyses.

A complete picture of the distribution of the occupied and unoccupied electronic states at the interface can be obtained by combining these direct photoemission spectra with inverse photoemission (IPES) results. Themlin et al. ${ }^{36}$ have indeed measured the conduction-band dispersion of a $1 \mathrm{ML} \mathrm{C}_{60}$ film on $\mathrm{GeS}(001)$. Comparing the intensity of the substrate diffraction spots in their published LEED picture to our results, we estimate that their actual $\mathrm{C}_{60}$ coverage was about $0.6 \mathrm{ML}$ rather than 1 ML. The evolution of the distribution of the occupied and unoccupied electronic states is shown in Fig. 20, for the clean substrate, a $0.6 \mathrm{ML} \mathrm{C}_{60}$ film, and a thick film. It is immediately apparent that the unoccupied states are more perturbed by the GeS substrate than the occupied ones. The features in the 0.6 ML IPES spectra are much less resolved and show a larger shift towards the Fermi
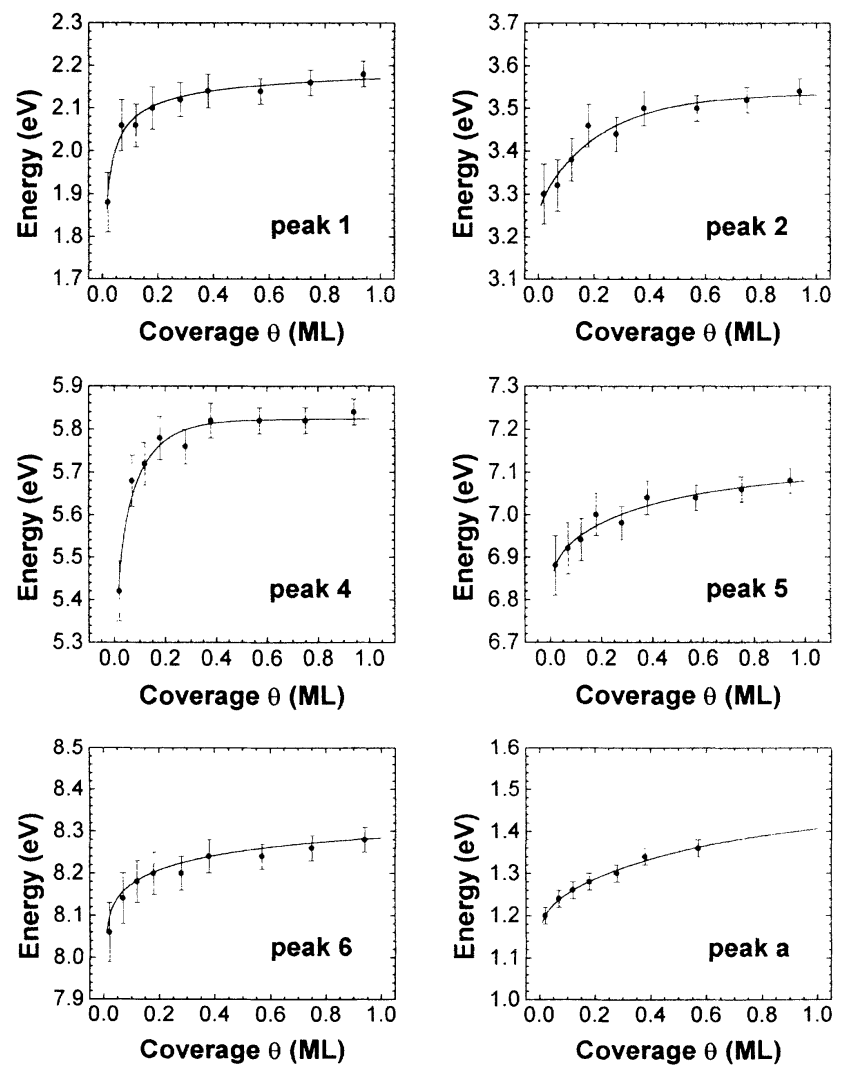

FIG. 19. Binding energy of the most intense $C_{60}$ features and of the first GeS valence-band peak versus coverage.

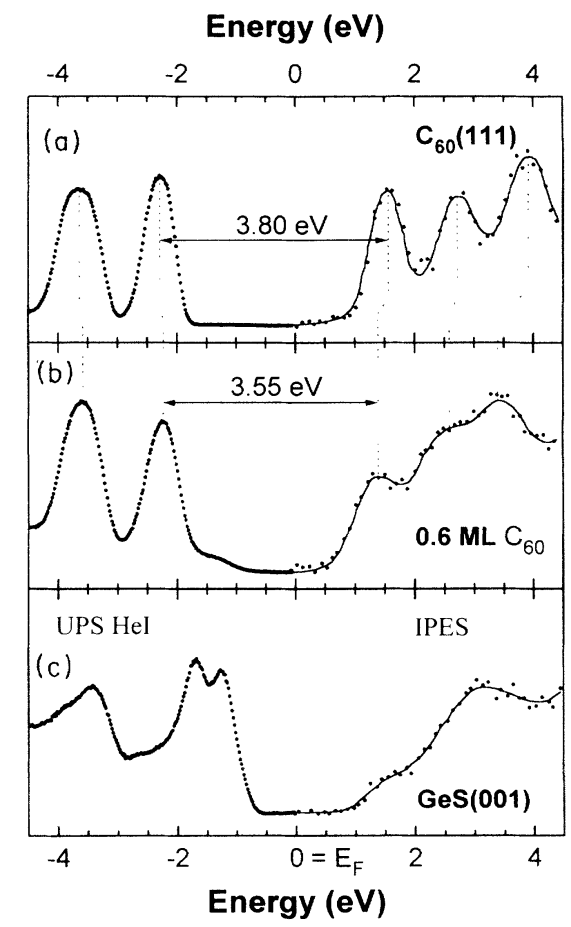

FIG. 20. Distribution of the occupied and unoccupied electronic states as probed by direct and inverse photoemission for (a) clean $\mathrm{GeS},(\mathrm{b}) \sim 0.6 \mathrm{ML} \mathrm{C}_{60}$, and (c) bulk $\mathrm{C}_{60}$.

level than the corresponding features in the UPS spectra. The shift depends on the molecular orbital under consideration: it is about $150 \mathrm{meV}$ for the LUMO- AND (LUMO + 1)-derived features, and reaches $500 \mathrm{meV}$ for the (LUMO+2)-derived feature. The larger perturbation of the higher-lying final states might reflect the larger spatial extension of the latter, which favors hybridization with the substrate, leading to the formation of a distribution of hybrid states.

The total effect of the shifts in the occupied and unoccupied features is a reduction of the measured HOMOLUMO separation from 3.8 to $3.55 \mathrm{eV}$ when going from the submonolayer regime to the solid. The observed gap reduction of $0.25 \mathrm{eV}$ is considerably smaller than that for $\mathrm{C}_{60}$ adsorption on metals [a value of $0.5 \mathrm{eV}$ has been reported for $\mathrm{C}_{60} / \mathrm{Au}$ (Ref. 29)]. This reflects the small interaction between the unoccupied $\mathrm{C}_{60}$ and $\mathrm{GeS}$ substrate levels compared to the stronger hybridization observed between the LUMO and the filled metal $d$ states.

\section{Work function measurements}

Adsorption of molecules on a solid surface in general modifies the sample work function. For a semiconductor a change in work function results from two contributions: ${ }^{42}$ (1) an adsorbate-induced band bending change $e \Delta V_{s}$, and (2) an interface dipole layer. The total work function change $e \Delta \phi$ is therefore given by

$$
e \Delta \phi=e \Delta \phi_{\mathrm{dip}}+e \Delta V_{s} .
$$

The dipole contribution to the change in work function $e \Delta \phi_{\text {dip }}$ is related to the dipole moment $p$ of the 
adsorbate/substrate complex, the density of adsorbed molecules or dipoles $n_{\text {dip }}$, and the polarizability $\alpha$ of the adsorbate/substrate complex by the equation ${ }^{42,56}$

$$
e \Delta \phi_{\mathrm{dip}}=-\frac{e}{\epsilon_{0}} p n_{\mathrm{dip}}\left(1+\frac{9 \alpha}{4 \pi} n_{\mathrm{dip}}^{3 / 2}\right)^{-1}
$$

where $\epsilon_{0}$ is the vacuum permittivity. The second term of the sum between parentheses, called the depolarization factor $f_{\text {dep }}$, is a correction to the work function change due to the interaction between the different dipoles which reduces the effective electric field at the site of a particular dipole. In the case of $\mathrm{C}_{60}$, due to the large size of the molecule the density of dipoles is quite small so that, in a first approximation, the interaction between the dipoles can be neglected in a wide range of values for the polarizability $\alpha$ of the dipole. Indeed, with a surface density of $n_{\mathrm{dip}}=1.15 \times 10^{14} \mathrm{C}_{60} / \mathrm{cm}^{2}$ [calculated assuming a fcc (111) structure with a lattice constant of $14.17 \AA$ for the first monolayer] and a polarizability $\alpha=65 \times 10^{-24} \mathrm{~cm}^{3}$ (Ref. 57) for an isolated $\mathrm{C}_{60}$ molecule, obtained from an $a b$ initio calculation, ${ }^{56}$ a value as small as 0.06 is obtained for the depolarization factor. Although the polarizability of the adsorbed $\mathrm{C}_{60}$ /substrate complex is not well known but probably larger than the gas-phase value, $f_{\text {dep }} \ll 1$ should be a good approximation. The dipole contribution to the work function change upon $\mathrm{C}_{60}$ adsorption can thus be written as

$$
e \Delta \phi_{\mathrm{dip}} \cong-\frac{e}{\epsilon_{0}} p n_{\mathrm{dip}} .
$$

A value for the size of the effective dipole $p$ can therefore be obtained from (5) and (7) if the total change in work function $e \Delta \phi$ and the band-bending change $e \Delta V_{s}$ are known. We have already measured that the adsorbateinduced band bending is about $0.2 \mathrm{eV}$.

In order to determine the work function change $e \Delta \phi$ upon $\mathrm{C}_{60}$ adsorption, we have recorded UPS spectra for increasing coverages. From the position $E_{c}$ of the cutoff on the high-binding-energy side (corresponding to the low-kinetic-energy side) the work function $e \phi$ can be calculated using

$$
e \phi=h v-E_{c},
$$

where $h v$ is the photon excitation energy. Figure 21(a) shows the high-binding-energy side of the $\mathrm{He} I$ $(h v=21.21 \mathrm{eV})$ excited photoemission spectrum for the $\mathrm{GeS}(001)$ substrate, $1 \mathrm{ML} \mathrm{C}_{60}$ adsorbed on $\mathrm{GeS}(001)$ and for a thick film representative of bulk fullerite. The position of the cutoff is determined as the zero of the second derivative. By coincidence, bulk fullerite and $\mathrm{GeS}(001)$ share the same work function value of $4.7 \mathrm{eV}$. A small but clear work function change of about $0.1 \mathrm{eV}$ is observed after adsorption of $1 \mathrm{ML}$ of $\mathrm{C}_{60}$. The complete evolution of the work function with increasing coverage is shown in Fig. 21(b). One observes a small increase in the work function with a maximum at around $1 \mathrm{ML}$ followed by a decrease and a saturation at the bulk value for $\mathrm{C}_{60}$ at around 2 or $3 \mathrm{ML}$. The position of the cutoff can be determined only within about $100 \mathrm{meV}$ because the
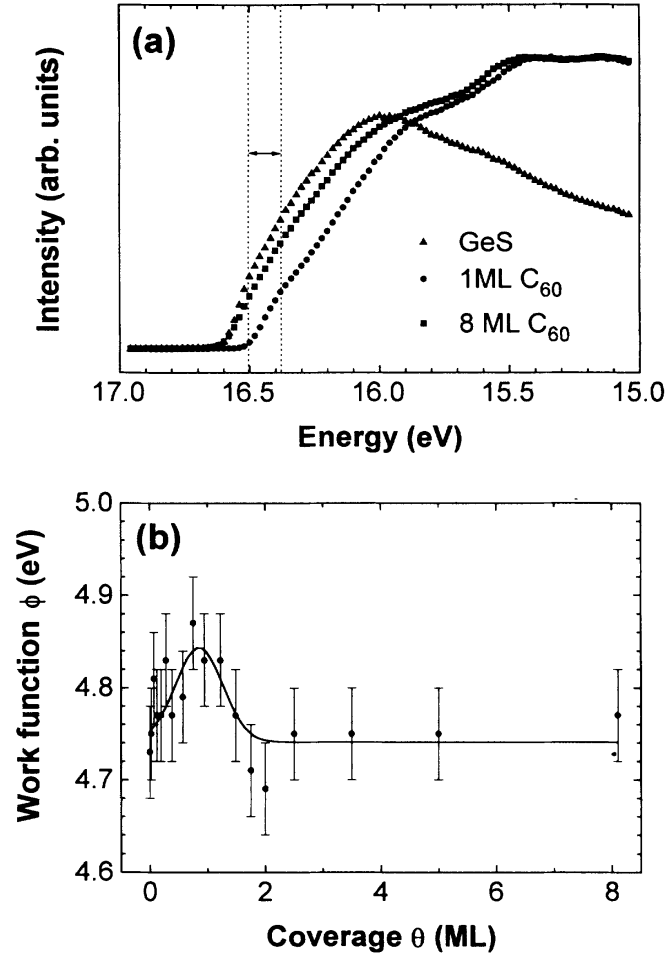

FIG. 21. (a) High-binding-energy side of the He I excited photoelectron spectra for $\operatorname{GeS}(001)(\Delta), 1 \mathrm{ML} \mathrm{C}_{60}(\bullet)$, and 8 $\mathrm{ML} \mathrm{C}_{60}(\boldsymbol{\square})$. The energies are referenced to the Fermi level of the sample. The work function increases from 4.7 to about 4.8 $\mathrm{eV}$ upon adsorption of $1 \mathrm{ML}$ of $\mathrm{C}_{60}$. (b) Plot of the sample work function versus $\mathrm{C}_{60}$ coverage. The solid line is the result of a least-squares Gaussian fit.

shape of the low-kinetic-energy side of the photoelectron spectra changes with coverage. This accounts for the large error bars in Fig. 21(b). Nevertheless, the experimental data points show a clear deviation to higher values at low coverages. The measured work function change $e \Delta \phi$ of about $0.1 \mathrm{eV}$ determined from a leastsquares Gaussian fit [shown as the solid line in Fig. 21(b)] is therefore acceptable although this value is of the same order of magnitude as the error bar.

According to Eq. (5) a work function increase of $0.1 \mathrm{eV}$ together with a downward band bending of $0.2 \mathrm{eV}$ give a dipole contribution of $-0.1 \mathrm{eV}$. Equation (6) yields then a value of $p \cong 8 \times 10^{-31} \mathrm{Cm}$ for the positive surface dipole. This value has to be compared to the estimated dipole moment of $6 \times 10^{-31} \mathrm{C} \mathrm{m}$ induced upon adsorption onto a metal due to the attractive image charge. This number is obtained as follows. Considering each carbon atom of the $\mathrm{C}_{60}$ molecule as an independently polarizable one-electron entity of static polarizability $\alpha$, its groundstate energy in the presence of the substrate is written as

$$
W(\delta)=\left\langle\Psi_{0}(z+\delta)\left|H_{0}+V\right| \Psi_{0}(z+\delta)\right\rangle,
$$

where

$$
V(z) \approx \frac{e^{2} \gamma}{4 z_{0}} \frac{3}{4} \frac{z^{3}}{z_{0}^{3}}
$$


is the dipolar interaction potential of the effective electron of coordinate $z$ normal to the surface with its selfimage in the substrate. $\gamma=(\epsilon-1) /(\epsilon+1)$ is the screening factor with $\epsilon$ the static dielectric function of the substrate; $z_{0}$ is the adsorption distance. In (9), $H_{0}$ is the free-atom Hamiltonian and $\left|\Psi_{0}(z+\delta)\right\rangle$ is taken to be its free ground state rigidly deplaced by an amount $\delta$ towards the surface. Taking $H_{0}$ and $\left|\Psi_{0}\right\rangle$ from a simple harmonic-oscillator model (the result obtained is not very sensitive to the one-effective-electron model) and minimizing $W(\delta)$ with respect to $\delta$, one finds the induced dipole moment per carbon atom

$$
p_{c}=e \delta=\frac{9}{32} \frac{\alpha}{z_{0}^{3}} \frac{e \gamma}{\beta^{2} z_{0}},
$$

where $\beta^{-1}$ is a measure of the carbon atomic radius [the Gaussian width of the ground-state wave function $\left.\sim \exp \left(-\beta^{2} z^{2} / 2\right)\right]$. The total dipole moment induced on the $\mathrm{C}_{60}$ molecule is obtained by summing (11) over the $\mathrm{C}_{60}$ carbon atoms. With $\alpha \sim 1 \AA^{3}, \beta \sim 1 \AA^{-1}$, and $\gamma \sim 1$ one obtains the value of $6 \times 10^{-31} \mathrm{C} \mathrm{m}$ mentioned above. For a semiconductor this induced dipole moment has to be reduced by the screening factor $\gamma \sim(\epsilon-1) /(\epsilon+1)$, which for $\mathrm{GeS}$ amounts to $\gamma \sim 0.86(\epsilon \sim 13)$. The difference between the calculated and measured surface dipole might be attributed to an additional polarization induced by the electric field of the ionic substrate. Calculations are in progress $^{45}$ in order to evaluate the magnitude of this contribution.

\section{CONCLUSION}

In this paper we have studied the formation of the $\mathrm{C}_{60} / \mathrm{GeS}(001)$ interface. We have presented evidence that the epitaxial $\mathrm{C}_{60}$ grows in a layer-by-layer mode. Highresolution TEM images have revealed a very low density of defects as well as a grain size larger than $1 \mu \mathrm{m}$. $\mathrm{GeS}(001)$ can therefore be considered as one of the best substrates for growing single-crystal $\mathrm{C}_{60}$ films; this is confirmed by recent angle-resolved photoemission measurements performed on these films. ${ }^{58}$ The investigation of the electronic structure of the interface by XPS and UPS has revealed that the overlayer-substrate interaction is mainly dominated by van der Waals forces. The formation of a weak but measurable interface dipole has been observed. This interface dipole has been interpreted in terms of an image-charge-induced polarization and receives an additional contribution from the static electric field generates by the cations and anions of the substrate.

\section{ACKNOWLEDGMENTS}

We thank G. Sawatzky for providing the $\mathrm{C}_{60}$ powder. We acknowledge fruitful discussions with J.-M. Gilles, Ph. Lambin, P. Rudolf, J.-M. Themlin, and J.-P. Vigneron. This work was supported by the Belgian National Program of Interuniversity Research Projects, the Wallonia Region, the European Community (Grant No. II91-15-EC), and the BMFT under Project No. 05 5GUABI.
${ }^{1}$ J. E. Rowe, P. Rudolf, L. H. Tjeng, R. A. Malic, G. Meigs, C. T. Chen, J. Chen, and E. W. Plummer, Int. J. Mod. Phys. B 6, 325 (1992).

${ }^{2}$ T. Hashizume, K. Motai, X. D. Wang, H. Shinohara, Y. Saito, Y. Maruyama, K. Ohno, Y. Kawazoe, Y. Nishina, H. W. Pickering, Y. Kuk, and T. Sakurai, Phys. Rev. Lett. 71, 2959 (1993).

${ }^{3}$ K. Motai, T. Hashizume, H. Shinohara, Y. Saito, H. W. Pickering, Y. Nishina, and T. Sakurai, Jpn. J. Appl. Phys. 32, L450 (1993).

${ }^{4}$ J. K. Gimzewski, S. Modesti, and R. R. Schlittler, Phys. Rev. Lett. 72, 1036 (1994).

${ }^{5} \mathrm{P}$. Rudolf et al. (private communication).

${ }^{6}$ E. I. Altman and R. J. Colton, Surf. Sci. 279, 49 (1992).

${ }^{7}$ E. I. Altman and R. J. Colton, Phys. Rev. B 48, 18244 (1993).

${ }^{8}$ J. K. Gimzewski, S. Modesti, Ch. Gerber, and R. R. Schlittler, Chem. Phys. Lett. 213, 401 (1992).

${ }^{9}$ Y. Kuk, D. K. Kim, Y. D. Suh, K. H. Park, H. P. Noh, S. J. Oh, and S. K. Kim, Phys. Rev. Lett. 70, 1948 (1993).

${ }^{10}$ X.-D. Wang, T. Hashizume, H. Shinohara, Y. Saito, Y. Nishina, and T. Sakurai, Phys. Rev. B 47, 15923 (1993).

${ }^{11}$ H. Xu, D. M. Chen, and W. N. Creager, Phys. Rev. Lett. 70, 1850 (1993).

${ }^{12}$ A. V. Hamza and M. Balooch, Chem. Phys. Lett. 201, 404 (1993).

${ }^{13}$ Y. Z. Li, J. C. Patrin, M. Chander, J. H. Weaver, L. P. F. Chibante, and R. E. Smalley, Science 252, 547 (1991).

${ }^{14}$ Y. Z. Li, M. Chander, J. C. Patrin, J. H. Weaver, L. P. F. Chibante, and R. E. Smalley, Science 253, 429 (1991).
${ }^{15}$ B. J. Benning, F. Stepniak, and J. H. Weaver, Phys. Rev. B 48, 9086 (1993).

${ }^{16}$ Y. Z. Li, M. Chander, J. C. Patrin, J. H. Weaver, L. P. F. Chibante, and R. E. Smalley, Phys. Rev. B 45, 13837 (1992).

${ }^{17}$ J. H. Weaver, Acc. Chem. Res. 25, 143 (1992).

${ }^{18}$ G. Gensterblum, L.-M. Yu, J.-J. Pireaux, P. A. Thiry, R. Caudano, J.-M. Themlin, S. Bouzidi, F. Coletti, and J.-M. Debever, Appl. Phys. A 56, 175 (1993).

${ }^{19}$ W. M. Tong, D. A. A. Ohlberg, H. K. You, R. S. Williams, S. J. Anz, M. M. Alvarez, R. L. Whetten, Y. Rubin, and F. N. Diederich, J. Phys. Chem. 95, 4709 (1991).

${ }^{20}$ H. Hong, W. E. McMahon, P. Zschack, D.-S. Lin, R. D. Aburano, H. Chen, and T. C. Chiang, Appl. Phys. Lett. 61, 3127 (1992).

${ }^{21}$ T. Chen, S. Howells, M. Gallagher, L. Yi, D. Sarid, D. L. Lichtenberger, K. W. Nebesny, and C. D. Ray, J. Vac. Sci. Technol. B 10, 170 (1992).

${ }^{22}$ H. P. Lang, V. Thommen-Geiser, J. Frommer, A. Zahab, P. Bernier, and H.-J. Güntherodt, Europhys. Lett. 18, 29 (1992).

${ }^{23}$ S. Behler, H. P. Lang, S. H. Pan, V. Thommen-Geiser, and H.-J. Güntherodt, Z. Phys. B (to be published).

${ }^{24}$ T. R. Ohno, Y. Chen, S. E. Harvey, G. H. Kroll, J. H. Weaver, R. E. Haufler, and R. E. Smalley, Phys. Rev. B 44, 13747 (1991).

${ }^{25}$ S. Modesti, S. Cerasari, and P. Rudolf, Phys. Rev. Lett. 71, 2469 (1993).

${ }^{26}$ A. J. Maxwell, P. A. Brühwiler, A. Nilsson, N. Martensson, and P. Rudolf, Phys. Rev. B 49, 10717 (1994).

${ }^{27}$ A. Sellidj and B. E. Koel, J. Phys. Chem. 97, 10076 (1993). 
${ }^{28}$ A. K. Santra, R. Seshadri, V. Vijayakrishnan, and C. N. R. Rao, Solid State Commun. 85, 77 (1993).

${ }^{29}$ T. R. Ohno, Y. Chen, S. E. Harvey, G. H. Kroll, P. J. Benning, J. H. Weaver, L. P. F. Chibante, and R. E. Smalley, Phys. Rev. B 47, 2389 (1993).

${ }^{30}$ R. L. Garrell, T. M. Herne, C. A. Szafranski, F. Diederich, F. Ettl, and R. L. Whetten, J. Am. Chem. Soc. 113, 6302 (1991).

${ }^{31}$ K. Akers, L. M. Cousins, and M. Moskovits, Chem. Phys. Lett. 190, 614 (1992).

${ }^{32}$ M. Sakurai, H. Tada, K. Saiki, and A. Koma, Jpn. J. Appl. Phys. 30, L565R (1991).

${ }^{33}$ K. Tanigaki, S. Kuroshima, J. Fujita, and T. W. Ebbesen, Appl. Phys. Lett. 63, 2351 (1993).

${ }^{34}$ M. Sakurai, H. Tada, K. Saiki, A. Koma, H. Funasaka, and Y. Kishimoto, Chem. Phys. Lett. 208, 425 (1993).

${ }^{35}$ G. Gensterblum, L.-M. Yu, J.-J. Pireaux, P. A. Thiry, R. Caudano, Ph. Lambin, A. A. Lucas, W. Krätschmer, and J. E. Fischer, J. Phys. Chem. Solids 53, 1427 (1992).

36J.-M. Themlin, S. Bouzidi, F. Coletti, J.-M. Debever, G. Gensterblum, L. M. Yu, J.-J. Pireaux, and P. A. Thiry, Phys. Rev. B 46, 15602 (1992).

${ }^{37}$ D. Schmicker, S. Schmidt, J. G. Skofronick, J. P. Toennies, and R. Vollmer, Phys. Rev. B 44, 10995 (1991).

${ }^{38}$ H. G. Busmann, R. Hiss, H. Gaber, and I. V. Hertel, Surf. Sci. 289, 381 (1993).

${ }^{39}$ W. Krakow, N. M. Rivera, R. A. Roy, R. S. Ruoff, and J. J. Cuomo, Appl. Phys. A 56, 185 (1993).

${ }^{40}$ J. E. Fischer, E. Werwa, and P. A. Heiney, Appl. Phys. A 56, 193 (1993).

${ }^{41}$ D. Bernaerts et al. (private communication).

${ }^{42}$ H. Lüth, Surfaces and Interfaces of Solids, Springer Series in Surface Sciences Vol. 15 (Springer-Verlag, Berlin, 1993).

${ }^{43}$ T. Grandke and L. Ley, Phys. Rev. B 16, 1616 (1977).

${ }^{44}$ P. A. Heiney, J. E. Fischer, A. R. McGhie, W. J. Romanow,
A. M. Denenstein, J. P. McCauley, and A. B. Smith, Phys. Rev. Lett. 66, 2911 (1991).

${ }^{45}$ A. A. Lucas (private communication).

${ }^{46} \mathrm{H}$. Betghe, in Interfacial Aspects of Phase Transformations, edited by B. Mutaftschiev (Reidel, Dordrecht, 1982), pp. 669-696.

${ }^{47}$ T. Yanagihara and H. Yamaguchi, Jpn. J. Appl. Phys. 23, 529 (1984).

${ }^{48}$ W. Allers, U. D. Schwarz, G. Gensterblum, and R. Wiesendanger, Appl. Phys. A 59, 11 (1994).

${ }^{49}$ C. Enkvist, S. Lunell, B. Sjörgen, S. Svensson, P. A. Brühwiler, A. Nilsson, A. J. Maxwell, and N. Martensson, Phys. Rev. B 48, 14629 (1993).

${ }^{50}$ T. Grandke and L. Ley, Phys. Rev. B 16, 832 (1977).

${ }^{51}$ M. Taniguchi, R. L. Johnson, J. Ghijsen, and M. Cardona, Phys. Rev. B 42, 3634 (1990).

${ }^{52}$ N. Troullier and J. L. Martins, Phys. Rev. B 46, 1754 (1992).

${ }^{53}$ P. J. Benning, F. Stepniak, D. M. Poirier, J. L. Martins, J. H. Weaver, L. P. F. Chibante, and R. E. Smalley, Phys. Rev. B 47, 13843 (1993).

${ }^{54}$ M. Merkel, M. Knupfer, M. S. Golden, J. Fink, P. Seemann, and R. L. Johnson, Phys. Rev. B 47, 11470 (1993).

${ }^{55}$ C. T. Chen, L. H. Tjeng, P. Rudolf, G. Meigs, J. E. Rowe, J. Chen, J. P. McCauley, Jr., A. B. Smith III, A. R. McGhie, W. J. Romanow, and E. W. Plummer, Nature 352, 603 (1991).

56J. R. MacDonald and C. A. Barlow, J. Chem. Phys. 39, 412 (1963).

${ }^{57}$ P. W. Fowler, P. Lazaretti, and R. Zanasi, Chem. Phys. Lett. 165, 79 (1990).

${ }^{58}$ G. Gensterblum, J. J. Pireaux, P. A. Thiry, R. Caudano, T. Buslaps, R. L. Johnson, G. Le Lay, V. Aristov, R. Günther, A. Taleb-Ibrahimi, G. Indlekofer, and Y. Petroff, Phys. Rev. B 48, 14756 (1993). 

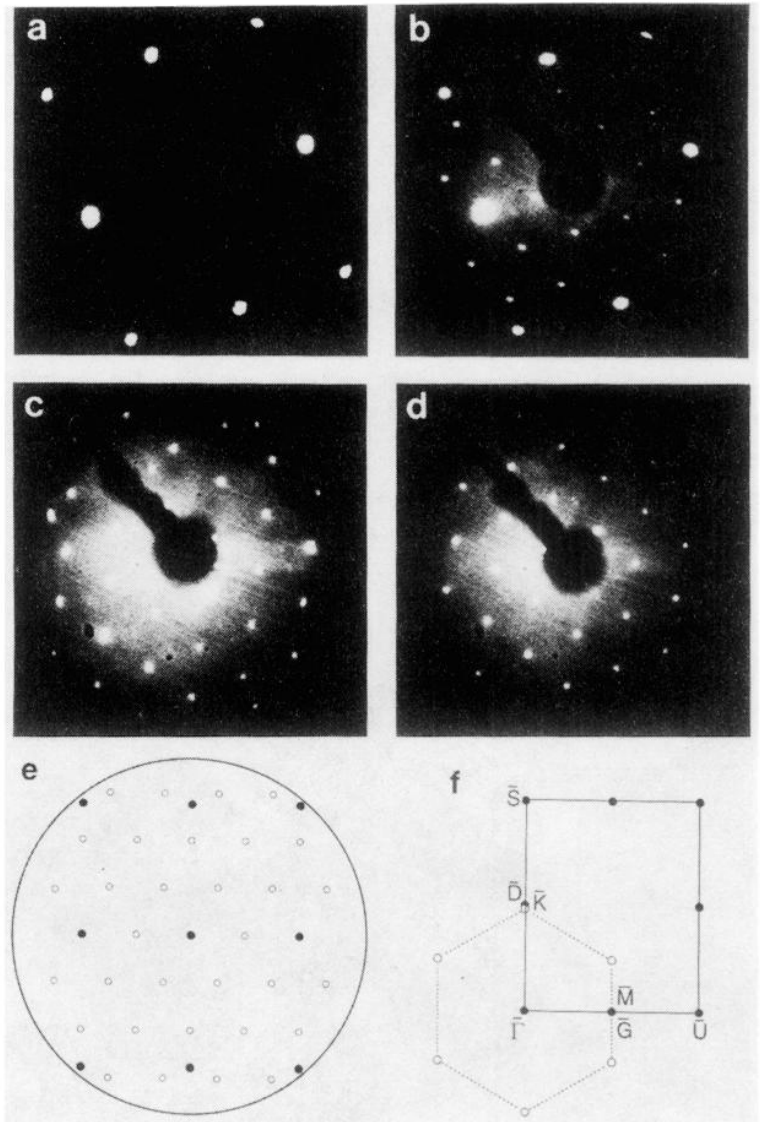

f

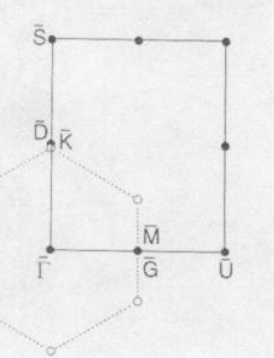

FIG. 1. LEED patterns recorded with $E_{p}=35 \mathrm{eV}$ of (a) a cleaved $\mathrm{GeS}(001)$ substrate; (b) $0.33 \mathrm{ML}$ and (c) $0.66 \mathrm{ML}$ of $\mathrm{C}_{60}$ on $\mathrm{GeS}$; both $\mathrm{C}_{60}$ and substrate spots are visible and they are identified by open and filled circles respectively in (e); (d) $1 \mathrm{ML}$ of $\mathrm{C}_{60}$ on $\mathrm{GeS}$; only spots of the fcc $\mathrm{C}_{60}$ (111) plane are visible; ( $f$ ) orientations of the first surface Brillouin zone of $\mathrm{C}_{60}(111)$ and the $\mathrm{GeS}(001)$ substrate: the $\bar{\Gamma}-\bar{K}(\bar{\Gamma}-\bar{M})$ direction of the $\mathrm{C}_{60}$ SBZ is parallel to the $\bar{\Gamma}-\bar{D}-\bar{S}(\bar{\Gamma}-\bar{G}-\bar{U})$ direction of the substrate SBZ. 


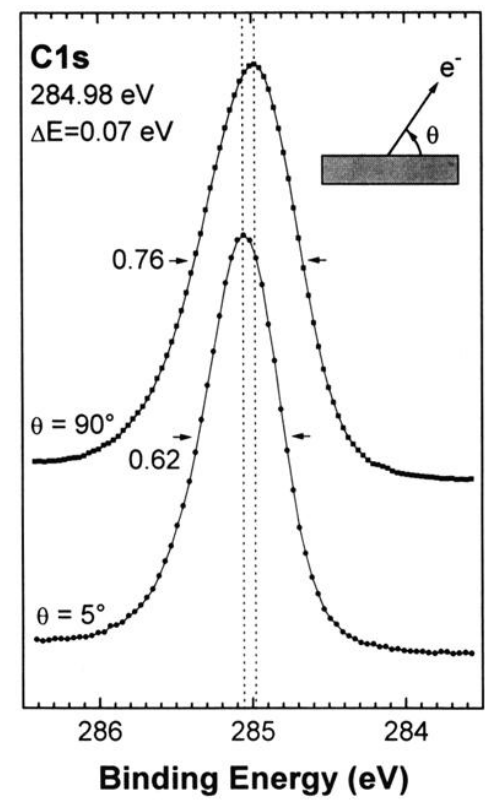

FIG. 12. C $1 s$ line shape for normal emission $\left(\theta=90^{\circ}\right)$ and for grazing incidence $\left(\theta=5^{\circ}\right)$. The grazing-emission spectrum is shifted to higher binding energies and has a smaller full width at half maximum $(0.62 \mathrm{eV}$ instead of $0.76 \mathrm{eV})$. 


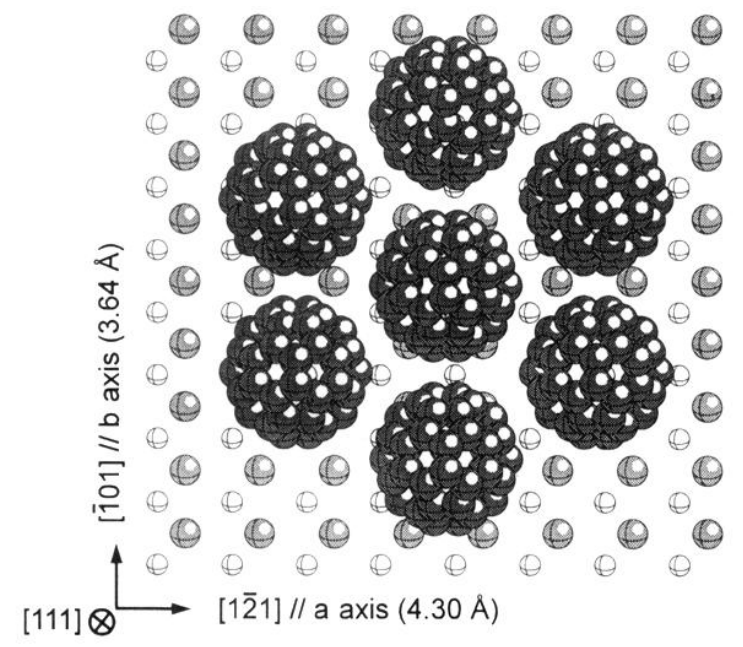

FIG. 2. Top view of the model structure of a $\mathrm{C}_{60}(111)$ layer grown on a $\mathrm{GeS}(001)$ substrate. Small grey (white) spheres represent the surface germanium (sulfur) atoms of $\mathrm{GeS}(001)$. The $\mathrm{C}_{60}[\overline{101}]([1 \overline{2} 1])$ direction is parallel to the $\mathrm{GeS} b$ axis $(a$ axis) so that the $\mathrm{C}_{60}(111)$ plane is parallel to the surface. 

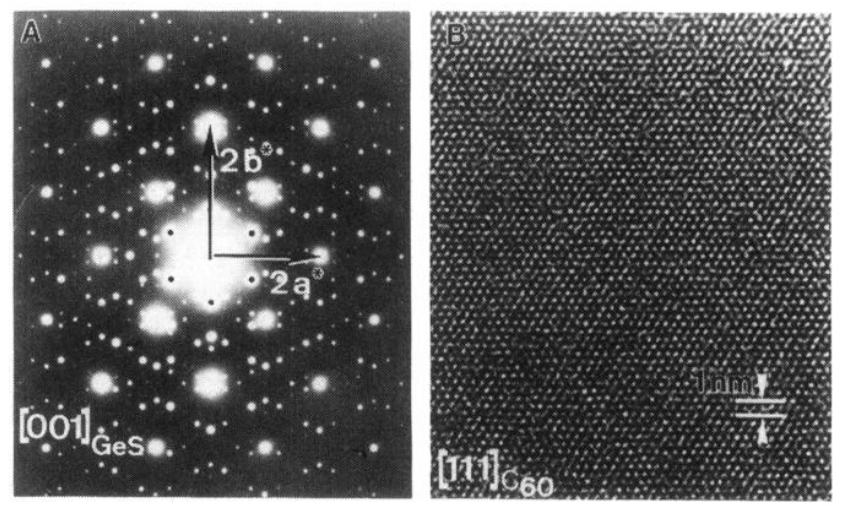

FIG. 3. (a) Selected-area diffraction pattern of a 200 - $\AA$-thick $\mathrm{C}_{60}$ film on $\mathrm{GeS}(001)$ along the [001] zone of the substrate. The reciprocal base vectors of the substrate are indicated; due to systematic extinctions the $\mathrm{IOO}$ and $\mathrm{OIO}$ ( $I$ odd) reflections are not visible. A schematic explanation of the complete diffraction pattern is given in Fig. 4. (b) High-resolution image of a $\mathrm{C}_{60}$ film on $\mathrm{GeS}(001)$ viewed along the [111] zone. Note the perfect structure of the film continuing over large areas. 


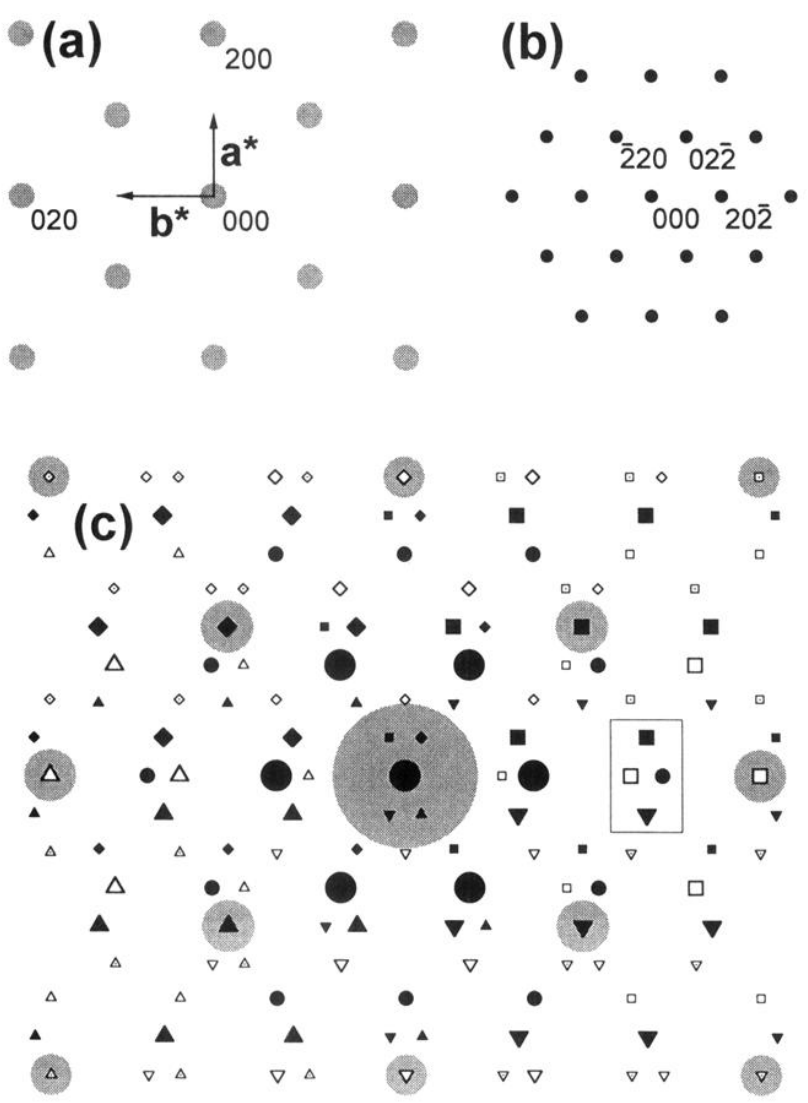

FIG. 4. Schematic diffraction patterns of (a) GeS(001) and (b) $\mathrm{C}_{60}(111)$. (c) Diffraction pattern of a thin $\mathrm{C}_{60}(111)$ film on $\mathrm{GeS}(001)$ obtained by superposing on each GeS diffraction spot a complete $\mathrm{C}_{60}[111]^{*}$ diffraction pattern. The different $\mathrm{C}_{60}$ $[111]^{*}$ diffraction patterns associated with each GeS spot are represented by different symbols. The size of the symbols decreases with increasing diffraction order so as to reproduce more or less the experimental intensities of the diffraction spots of Fig. 3(a). 


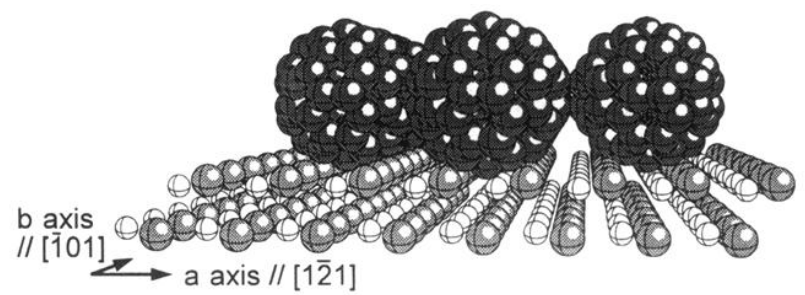

FIG. 5. Side view of the model structure of a $\mathrm{C}_{60}(111)$ monolayer on a $\mathrm{GeS}(001)$ substrate showing the peculiar corrugation in the $a$ direction of the basal plane and the systematic skipping of one interchain groove by the big $\mathrm{C}_{60}$ molecule. 